\title{
Homogeneous metallicities and radial velocities for Galactic globular clusters $\star$, $\star \star$
}

\section{New CaT metallicities for $\mathbf{2 8}$ distant and reddened globular clusters}

\author{
S. Vásquez ${ }^{1}$, I. Saviane ${ }^{2}$, E. V. Held ${ }^{3}$, G. S. Da Costa ${ }^{4}$, B. Dias², M. Gullieuszik ${ }^{3}$, B. Barbuy ${ }^{5}$, \\ S. Ortolani ${ }^{6,3}$, and M. Zoccali ${ }^{7,8}$
}

\author{
${ }^{1}$ Museo Interactivo Mirador, Dirección de Educación, Av. Punta Arenas 6711, La Granja, Santiago, Chile \\ 2 European Southern Observatory, Alonso de Córdova 3107, Santiago, Chile \\ 3 INAF - Osservatorio Astronomico di Padova, vicolo Osservatorio 5, 35122 Padova, Italy \\ e-mail: enrico.held@oapd.inaf.it \\ ${ }^{4}$ Research School of Astronomy \& Astrophysics, Australian National University, Mt Stromlo Observatory, via Cotter Rd, Weston, \\ ACT 2611, Australia \\ 5 Universidade de São Paulo, Rua do Matão 1226, São Paulo 05508-900, Brazil \\ 6 Dipartimento di Fisica e Astronomia, Università degli Studi di Padova, vicolo dell'Osservatorio 3, 35122 Padova, Italy \\ 7 Instituto de Astrofísica, Facultad de Física, Pontificia Universidad Católica de Chile, Av. Vicuña Mackenna 4860, Santiago, Chile \\ ${ }^{8}$ Millennium Institute of Astrophysics, Av. Vicuña Mackenna 4860, Santiago, Chile
}

Received 29 May 2018 / Accepted 25 July 2018

\begin{abstract}
Although the globular clusters in the Milky Way have been studied for a long time, a significant fraction of them lack homogeneous metallicity and radial velocity measurements. In an earlier paper we presented the first part of a project to obtain metallicities and radial velocities of Galactic globular clusters from multiobject spectroscopy of their member stars using the ESO Very Large Telescope. In this paper we add metallicities and radial velocities for a new sample of 28 globular clusters, including in particular globular clusters in the MW halo and the Galactic bulge. Together with our previous results, this study brings the number of globular clusters with homogeneous measurements to $\sim 69 \%$ of those listed in the Harris' catalogue. As in our previous work, we have used the Ca II triplet lines to derive metallicities and radial velocities. For most of the clusters in this study, this is the first analysis based on spectroscopy of individual member stars. The metallicities derived from the $\mathrm{Ca}$ II triplet are then compared to the results of our parallel study based on spectral fitting in the optical region and the implications for different calibrations of the Ca II triplet line strengths are discussed. We also comment on some interesting clusters and investigate the presence of an abundance spread in the globular clusters here. A hint of a possible intrinsic spread is found for NGC 6256, which therefore appears to be a good candidate for further study.
\end{abstract}

Key words. stars: abundances - stars: kinematics and dynamics - globular clusters: general

\section{Introduction}

Globular clusters (GCs) were considered as simple stellar populations for a long time, with most of their parameters derived from heterogeneous methods and data, largely driven by the technology available at the time when the clusters were studied. Recently, with the acquisition of extremely precise photometry from Hubble Space Telescope (HST) imagers and spectroscopy from large telescopes, the idea of simplicity has been replaced by a more complex view of their star forming history (see, e.g., Gratton et al. 2012). Closely linked to that history is the dynamical evolution of clusters, which is another area of study that benefits from homogeneous samples and reduction techniques. Data sets like these can be used as input to N-body simulations, and the resulting dynamical parameters allow comparative studies such as that recently published in Baumgardt \& Hilker (2018).

\footnotetext{
$\star$ Based on observations taken with ESO telescopes at the La Silla Paranal Observatory under programme ID 089.D-0493(B).

$\star \star$ Full Table 3 is only available at the CDS via anonymous $\mathrm{ftp}$ to cdsarc.u-strasbg. fr (130.79.128.5) or via http://cdsarc. u-strasbg.fr/viz-bin/qcat?J/A+A/619/A13
}

Improvements in our understanding of $\mathrm{GC}$ evolution came also from the introduction of large spectroscopic studies, which enabled measuring homogeneous abundances for large samples of GC stars. The largest sample of metallicities measured with the same method comes from the study of equivalent widths of the Ca II triplet (CaT) lines in the spectra of GC stars by Rutledge et al. (1997) who determined metallicities for 52 clusters and made a literature compilation on their scale for a total of 71 clusters. The second largest sample is represented by integrated-light spectroscopic studies such as those of Zinn \& West (1984, ZW84) and Armandroff \& Zinn (1988) which account for an additional $\sim 20 \%$ of the metallicity measurements for GCs. Another approach to metallicity homogenisation was followed by Carretta et al. (2009) using their high-resolution spectroscopic analysis of 19 GCs to set a new metallicity scale based on $\mathrm{Fe}$ I lines. Transformations to the new scale were given for 114 GCs from Harris (1996).

The lack of homogeneous measurements currently mostly affects the outer-halo (distant) and bulge (highly extincted) globular clusters, which give important constraints on the Milky Way formation. This motivated our group to increase the sample 
of GCs with metallicities determined in a consistent way. The project is based on medium-resolution spectroscopy of individual red giant branch (RGB) stars in a sample of clusters which complements that of Rutledge et al. (1997). The spectra were obtained with FORS2 at the ESO VLT observatory in two spectral intervals, the $\mathrm{CaT}$ region and the green region that is rich in metal absorption lines.

In Paper I (Saviane et al. 2012, S12) we presented the methods and analysis of spectra in the CaT region for a first set of clusters from the original sample, including eight calibration GCs and 20 programme clusters, four of which are in common with Rutledge et al. (1997). The use of spectra at the CaT is particularly useful for targets near the Galactic plane or towards the Milky Way bulge because of the lower extinction in the farred spectral range. A new CaT metallicity calibration based on the Carretta et al. (2009; hereafter C09) abundance scale was derived and used to measure the metallicity of the programme clusters. In addition, the clusters from Rutledge et al. (1997) were included, converted to the new scale.

In a parallel project, new metallicity measurements were obtained for a largely overlapping GC sample from full spectral fitting of medium resolution spectra in the visible region (Dias et al. 2015, 2016a). The new metallicity scale, independent of previous empirical calibrations, appears to be consistent both with abundances from high-resolution spectroscopy and photometric metallicities (Cohen 2017). On the basis of the new scale, and taking advantage of an increased sample of metal-rich clusters, Dias et al. (2016b) generated a homogeneous compilation of metallicities for $152 \mathrm{GCs}$ ( $97 \%$ of the total) from different sources.

This paper increases our homogeneous database by adding new CaT measurements for a further set of $28 \mathrm{GCs}$, which in combination with those published in Saviane et al. (2012) provide metallicity estimates for $\sim 69 \%$ of the Milky Way globular clusters in the Harris $(1996)^{1}$ catalogue. The $[\mathrm{Fe} / \mathrm{H}]$ estimates from the CaT are then compared to the GC metallicities independently obtained from the green spectral interval (Dias et al. 2015, 2016a).

This paper is organised as follows: in Sect. 2 the observations and data reduction are presented; in Sect. 3, radial velocities are measured and compared with literature values; in Sect. 4 several CaT EW calibration relations are discussed and metallicities are estimated. Section 5 provides comments for some interesting clusters. Finally, our results are summarised in Sect. 6.

\section{Observations and data reduction}

The complete cluster programme of this project contains 56 clusters, selected from the catalogue of Galactic globular clusters published by Harris (1996, version February 2003). The observations and data reduction followed the procedure described in Saviane et al. (2012), where we presented our CaT results for the clusters observed in ESO Period 77. In order to complete the project, observing time using FORS2 mounted on the UT1-Antu telescope was assigned in ESO P89. Observations were performed in MOS mode for all clusters, using 19 movable slitlets to obtain multislit spectra. The $1028 \mathrm{z}+29$ grism and the OG590+32 orderblocking filter were set, obtaining a spectral coverage $\sim 7700$ $9500 \AA$ at a scale of $0.85 \AA$ per (binned) pixel. The observing $\log$ is given in Table 1. As shown in Fig. 1, the clusters analysed in this work cover the faint and redder part of our full cluster sample. A target list of RGB stars was selected in a narrow

\footnotetext{
1 Hereafter all references to the catalogue refer to the 2010 on-line version (Harris 2010), unless otherwise specified.
}

Table 1. Observing log including exposure time, reddening and metallicity source for the 28 programme clusters.

\begin{tabular}{llll}
\hline \hline Cluster & $t_{\exp }(\mathrm{s})$ & $E(B-V)$ & {$[\mathrm{Fe} / \mathrm{H}]$} \\
\hline BH 176 & $1 \times 240$ & 0.54 & (Phot)/PS03 \\
Djorg 1 & $2 \times 1560$ & 1.58 & (Phot)/VFO10 \\
Djorg 2 & $1 \times 60$ & 0.94 & (Phot)/VFO10 \\
NGC 5634 & $1 \times 60$ & 0.05 & (i)/ZW84 (Q39) \\
NGC 5694 & $1 \times 240$ & 0.09 & (i)/AZ88, LLC06 \\
NGC 5946 & $1 \times 60$ & 0.54 & (i)/AZ88 \\
NGC 6256 & $1 \times 360$ & 1.09 & (i)/SF04 \\
NGC 6284 & $1 \times 60$ & 0.28 & (i)/ZW84 (Q39) \\
NGC 6316 & $1 \times 60$ & 0.54 & (i)/AZ88 \\
NGC 6352 & $1 \times 60$ & 0.22 & (h)/F91, FPJ09 \\
NGC 6355 & $1 \times 120$ & 0.77 & (i)/ZW84 (Q39) \\
NGC 6366 & $1 \times 60$ & 0.71 & (CaT)/DA95, DS89 \\
NGC 6401 & $1 \times 120$ & 0.72 & (Fe)/M95a \\
NGC 6426 & $1 \times 200$ & 0.36 & (i)/ZW84 (Q39) \\
NGC 6453 & $1 \times 120$ & 0.64 & (h)/VFO10 \\
NGC 6517 & $1 \times 420$ & 1.08 & (Fe)/M95a \\
NGC 6539 & $1 \times 180$ & 1.02 & (i)/SF04, O\&\&05 \\
NGC 6749 & $1 \times 780$ & 1.50 & (Phot)/KHM97 \\
NGC 6864 & $90 / 180 / 60$ & 0.16 & (h)/Car09 \\
Pal 10 & $1 \times 660$ & 1.66 & (Phot)/KH97 \\
Pal 11 & $60 / 180$ & 0.35 & (CaT)/ADZ92, DA95 \\
Pal 14 & $1 \times 1100$ & 0.04 & (CaT)/ADZ92 \\
Pal 6 & $1 \times 550$ & 1.46 & (h)/LC02, SF04, LCB04 \\
Terzan 1 & $1 \times 1200$ & 1.99 & (i)/AZ88, I\&\&02 \\
Terzan 2 & $1 \times 1100$ & 1.87 & (i)/AZ88, SF04 \\
Terzan 8 & $3 \times 120$ & 0.12 & (CaT)/DA95, MWM08 \\
Terzan 9 & $1100 / 100$ & 1.76 & (Phot)/VFO10 \\
Ton 2 & $1 \times 360$ & 1.24 & (Phot)/BOB96 \\
\hline
\end{tabular}

Notes. The metallicity source from Harris catalogue (Harris 1996) is coded as (method)/references, where the reference acronym can be found in the catalogue bibliography list. The methods are (h)igh resolution, (i)ntegrated light, medium-resolution EW of (Fe) lines, (CaT) method or (Phot)ometric studies.

range from 1 mag below to 3 mag above the horizontal branch (HB) from colour-magnitude diagrams (CMDs) obtained from the pre-imaging observations in the $V, I$ bands, and used to define the MOS slitlets configuration. To this aim, we set a magnitude threshold depending on the cluster and generally fainter than the limit $V=20$ used in Saviane et al. (2012).

All the spectra were extracted using the FORS2 pipeline version 4.9. The pipeline reduces each target spectrum using daytime calibration frames to compute a wavelength calibration and distortion correction for each slit. The wavelength calibration is improved by aligning the solutions to the position of the sky lines (skyalign option). The individual spectra were corrected for bias, flat field and local sky background. The final spectra were extracted using the Horne (1986) optimal extraction and average combined (after radial velocity correction) when more than one spectrum was observed.

\section{Radial velocities}

Heliocentric radial velocities were measured for each spectrum using the IRAF fxcor task to perform cross-correlation between the target spectrum and a template spectrum with known radial velocity. This task provides observed radial velocities and heliocentric corrections using the celestial coordinates 


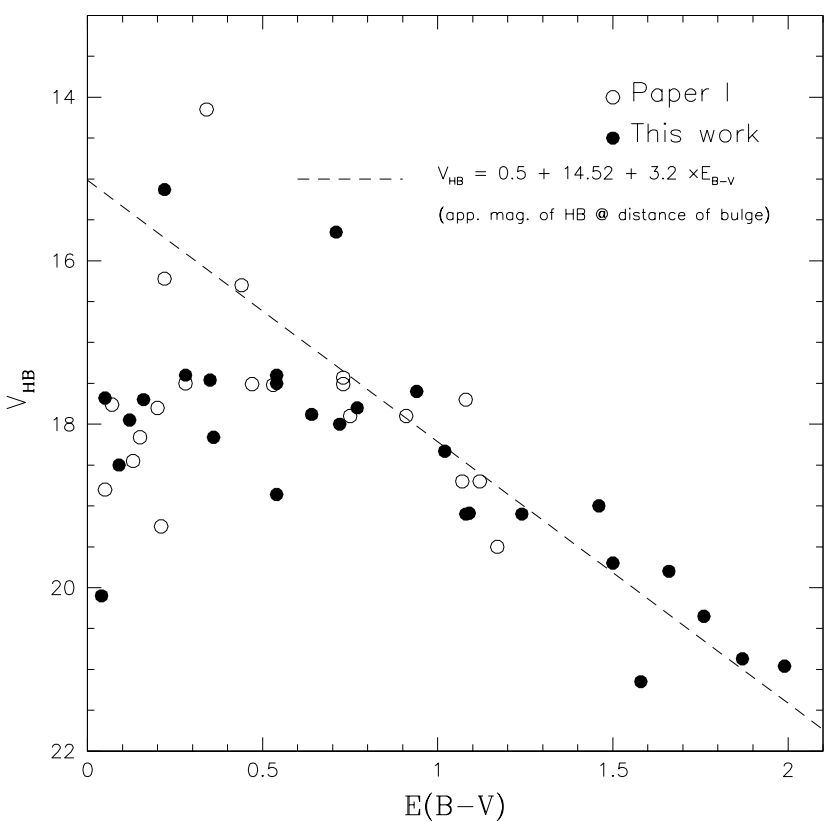

Fig. 1. Reddening and visual apparent magnitude at the level of the horizontal branch for the globular clusters observed in Saviane et al. (2012) and this work. Data are from the Harris catalogue. The dashed line shows the $V$ magnitude of the horizontal branch (assuming $V_{0, \mathrm{HB}}=0.5$ ) for a cluster at the distance of the bulge $(8 \mathrm{kpc})$ and subject to different degrees of extinction.

of the field and the observatory position. The cross-correlation was done against a single synthetic spectrum for a typical metalpoor K giant star with $T_{\text {eff }}=4750 \mathrm{~K}, \log g=2.5$, and $[\mathrm{Fe} / \mathrm{H}]=$ -1.3 covering the CaT region from $8450 \AA$ to $8750 \AA$. Using the selection criteria presented in Saviane et al. (2012), candidate member stars were selected under the assumption that stars in a cluster have similar radial velocities and small dispersion of the order $\sim 10 \mathrm{~km} \mathrm{~s}^{-1}$ (Pryor \& Meylan 1993). To define a first guess mean radial velocity for member selection, a $3 \sigma$ clipping iteration was used for all stars in each cluster using the median and the first quartile as estimators. After this pre-selection, the sum of the strengths of the $\lambda 8542$ and $\lambda 8662$ lines of the CaT was plotted against $V-V_{\mathrm{HB}}$. In this plot, stars with similar metallicities are distributed along a line with fixed slope (see next section). Data points deviating from the regression line defined by the median of candidate members were excluded by a new $3 \sigma$ clipping iteration. As an example, Fig. 2 shows the selection process for the cluster NGC 5946.

The heliocentric radial velocities in this work were compared with those measured by our group in the visual spectral range 4560-5860 $\AA$ (Dias et al. 2016a; hereafter D16). The radial velocities of $34 \mathrm{GCs}$ in common with the CaT sample (including the results of Saviane et al. 2012) are compared in Fig. 3. The two independent sets of measurements show a small systematic offset $\left(v_{\mathrm{CaT}}-v_{\mathrm{D} 16}=-9.2 \mathrm{~km} \mathrm{~s}^{-1}\right)$. The radial velocity is more accurate in the CaT region because of the larger number of sky lines used to correct the wavelength calibration. Our measurements are also compared in Fig. 4 with radial velocities published for 26 clusters in the Harris (1996) catalogue, which are weighted averages of data collected from various published sources.

The mean difference is $-4.5 \mathrm{~km} \mathrm{~s}^{-1}$ with a standard deviation $24 \mathrm{~km} \mathrm{~s}^{-1}$, a shift and dispersion that mostly originate from ten clusters with uncertainties larger than $8 \mathrm{~km} \mathrm{~s}^{-1}$ in the catalogue. In view of the homogeneous data quality and reduction in our work,

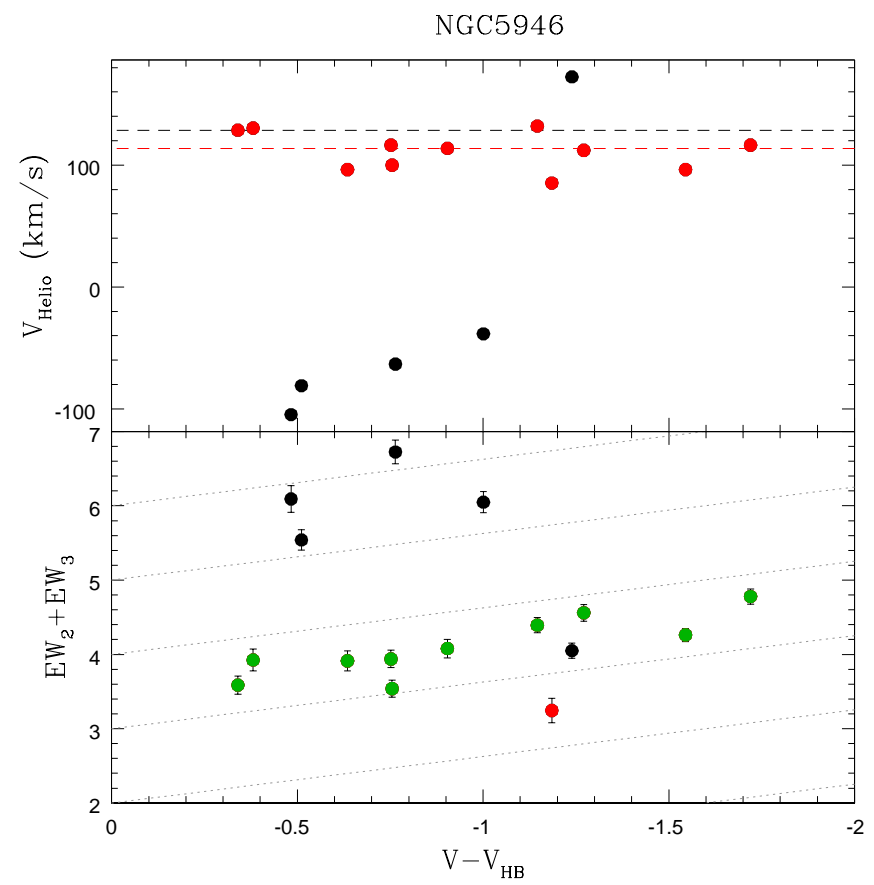

Fig. 2. Cluster member selection for NGC 5946. The upper panel shows in red the stars selected on the basis of radial velocity criteria, while black dots represent non-members. The mean radial velocity derived from the selected stars is shown as a red dashed line. This value is $\sim 15 \mathrm{~km} \mathrm{~s}^{-1}$ lower than the radial velocity in the Harris (1996) catalogue (black dashed line). Lower panel: selection based on the line strength of the $\mathrm{Ca}$ II triplet lines. For member selection we used isoabundance lines with a fixed slope as in Saviane et al. (2012; dotted lines). The green dots are stars accepted as cluster members on the basis of both criteria. Only one radial-velocity member was excluded from the sample because of its line strength (red dot).

we believe our values are systematically more accurate. Indeed, by picking only GCs with small uncertainties in the Harris' catalogue $\left(<2 \mathrm{~km} \mathrm{~s}^{-1}\right)$, the mean and standard deviation of the difference are reduced to $-0.7 \mathrm{~km} \mathrm{~s}^{-1}$ and $10 \mathrm{~km} \mathrm{~s}^{-1}$ respectively. A compilation of radial velocity data for the GCs analysed here is given in Table 2, which, together with Table 2 in Saviane et al. (2012), provides a homogeneous radial velocity data set for faint and reddened Galactic globular clusters.

\section{Metallicity measurements}

\subsection{Equivalent width measurements}

The EWs were measured with the methods described in Vásquez et al. (2015). As in Paper I, we used the sum of the EWs of the two strongest CaT lines $(\lambda 8542, \lambda 8662)$ as a metallicity estimator, following the Ca II triplet method of Armandroff \& Da Costa (1991). Different functions have been tested in the literature to measure the EWs of the CaT lines, depending on the metallicity regime. For metal-poor stars $([\mathrm{Fe} / \mathrm{H}] \lesssim-0.7 \mathrm{dex})$ a Gaussian function was used with excellent results (Armandroff \& Da Costa 1991), while a more general function (such as a Moffat function or the sum of a Gaussian and Lorentzian, $G+L$ ) is needed to fit the strong wings of the CaT lines observed in metal-rich stars (Rutledge et al. 1997; Cole et al. 2004). Following our previous work (Gullieuszik et al. 2009; Saviane et al. 2012) we have adopted here a G+L profile fit. To measure the EWs, each spectrum was normalised with a low-order polynomial using the IRAF continuum task, and set 


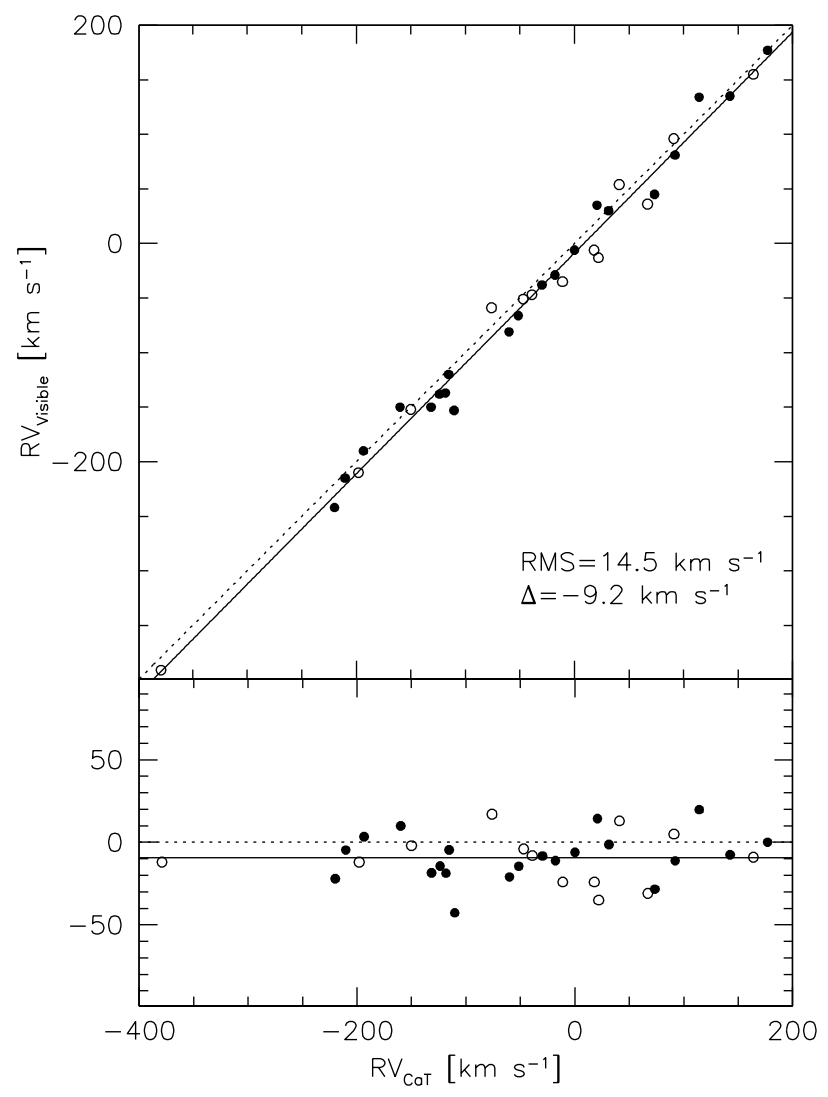

Fig. 3. Comparison between radial velocities for globular clusters analysed in Saviane et al. (2012; open circles) and this work (filled circles), and the measurements of Dias et al. (2016a). Upper panel: the dotted line refers to the one-to-one relation, while the continuous line is a linear fit to the data. In the lower panel we plot the residuals $v_{\mathrm{vis}}-v_{\mathrm{CaT}}$

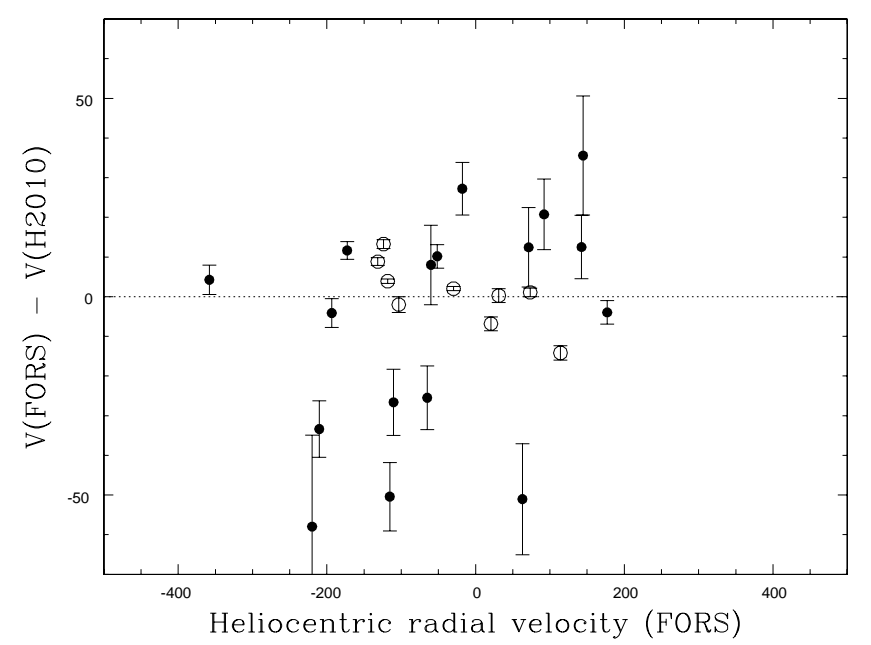

Fig. 4. Radial velocity comparison between our measurements and those listed by Harris (1996). The open circles are clusters with errors smaller than $2 \mathrm{~km} \mathrm{~s}^{-1}$ in the Harris' catalogue, while the filled circles mark clusters with errors larger than $2 \mathrm{~km} \mathrm{~s}^{-1}$.

to the rest frame by correcting for the observed radial velocity. The two strongest CaT lines were fitted by a $\mathrm{G}+\mathrm{L}$ profile using a non-linear least squares fitting routine part of the $\mathrm{R}$ programming language. Five clusters from the sample of Saviane et al. (2012) covering a wide metallicity range were re-reduced and analysed with our code to ensure that our EWs measurements are on the same scale as the template clusters used to define the metallicity
Table 2. Mean radial velocities of globular clusters.

\begin{tabular}{|c|c|c|c|c|c|c|}
\hline Cluster & $N$ & $v_{\mathrm{r}}$ & Err & $\begin{array}{r}v_{\mathrm{r}} \\
\text { (lit.) }\end{array}$ & Err & $\Delta v_{\mathrm{r}}$ \\
\hline BH 176 & 11 & 0.1 & 0.7 & 0.0 & 15 & 0.1 \\
\hline Djorg 1 & 3 & -358.1 & 0.7 & -362.4 & 3.6 & 4.2 \\
\hline Djorg 2 & 3 & -159.9 & 0.5 & & & \\
\hline NGC 5634 & 10 & -17.9 & 0.4 & -45.1 & 6.6 & 27.2 \\
\hline NGC 5694 & 9 & -131.5 & 0.6 & -140.3 & 0.8 & 8.8 \\
\hline NGC 5946 & 10 & 114.2 & 0.4 & 128.4 & 1.8 & -14.2 \\
\hline NGC 6256 & 10 & -103.4 & 0.5 & -101.4 & 1.9 & -2.0 \\
\hline NGC 6284 & 7 & 20.7 & 0.4 & 27.5 & 1.7 & -6.8 \\
\hline NGC 6316 & 3 & 92.2 & 0.6 & 71.4 & 8.9 & 20.8 \\
\hline NGC 6352 & 12 & -123.7 & 0.3 & -137.0 & 1.1 & 13.3 \\
\hline NGC 6355 & 7 & -210.3 & 0.4 & -176.9 & 7.1 & -33.4 \\
\hline NGC 6366 & 14 & -118.3 & 0.3 & -122.2 & 0.5 & 3.9 \\
\hline NGC 6401 & 7 & -115.4 & 0.3 & -65.0 & 8.6 & -50.4 \\
\hline NGC 6426 & 7 & -220.0 & 1.0 & -162.0 & 23.0 & -58.0 \\
\hline NGC 6453 & 6 & -110.3 & 0.5 & -83.7 & 8.3 & -26.6 \\
\hline NGC 6517 & 9 & -65.1 & 0.2 & -39.6 & 8.0 & -25.5 \\
\hline NGC 6539 & 9 & 31.3 & 0.4 & 31.0 & 1.7 & 0.3 \\
\hline NGC 6749 & 8 & -51.5 & 0.7 & -61.7 & 2.9 & 10.2 \\
\hline NGC 6864 & 11 & -193.4 & 0.2 & -189.3 & 3.6 & -4.1 \\
\hline Pal 10 & 9 & -29.7 & 0.3 & -31.7 & 0.4 & 2.0 \\
\hline Pal 11 & 7 & -60.0 & 0.4 & -68.0 & 10.0 & 8.0 \\
\hline Pal 14 & 5 & 73.4 & 1.1 & 72.3 & 0.2 & 1.1 \\
\hline Pal 6 & 3 & 177.0 & 1.0 & 181.0 & 2.8 & -4.0 \\
\hline Terzan 1 & 9 & 63.0 & 0.5 & 114.0 & 14.0 & -51.0 \\
\hline Terzan 2 & 3 & 144.6 & 0.8 & 109.0 & 15.0 & 35.6 \\
\hline Terzan 8 & 7 & 142.5 & 0.4 & 130.0 & 8.0 & 12.5 \\
\hline Terzan 9 & 6 & 71.4 & 0.4 & 59.0 & 10.0 & 12.4 \\
\hline Ton 2 & 8 & -172.7 & 0.3 & -184.4 & 2.2 & 11.7 \\
\hline
\end{tabular}

Notes. Column 2 gives the number of member stars used to determine the mean radial velocity that is listed in Col. 3. Column 4 lists the standard error of the mean. The radial velocities in the literature (Cols. 5 and 6) are from the Harris catalogue, except for $\mathrm{BH} 176$, for which the source is Sharina et al. (2014). The difference $\Delta v_{\mathrm{r}}$ is given in the last column as FORS2-H10.

calibration. Figure 5 shows the comparison between our EWs measurements and the line strengths measured by Saviane et al. (2012) (in both cases the sum of the two strongest lines) for the five calibration clusters. The observed scatter is consistent with the internal errors of the EW measurements, computed as in Vásquez et al. (2015). The measurements show a small deviation from the unity relation, which is more evident at low metallicity. A linear fit to this trend gives the relation: $\Sigma_{\mathrm{EW}}(\mathrm{S} 12)=0.97 \Sigma_{\mathrm{EW}}$ (this work) +0.21 , with an rms about the fit of $0.13 \AA$. This fit is shown in Fig. 5 as a dashed black line. For internal consistency, all EWs in this work have been adjusted to the measurement scale of Saviane et al. (2012) by using this relation. In Table 3 we provide the coordinates, radial velocities, and the sum of the equivalent widths for the cluster member stars, both measured ("m") and corrected ("c") to the system of Saviane et al. 2012.

\subsection{Reduced equivalent widths}

According to the CaT method, the dependence on the stellar gravity and $T_{\text {eff }}$ can be empirically corrected within a cluster by defining the reduced equivalent width $W^{\prime}$ as

$W^{\prime}=\Sigma_{\mathrm{EW}}-\beta\left(V-V_{\mathrm{HB}}\right)$, 
S. Vásquez et al.: Homogeneous metallicities and radial velocities for Galactic globular clusters. II.

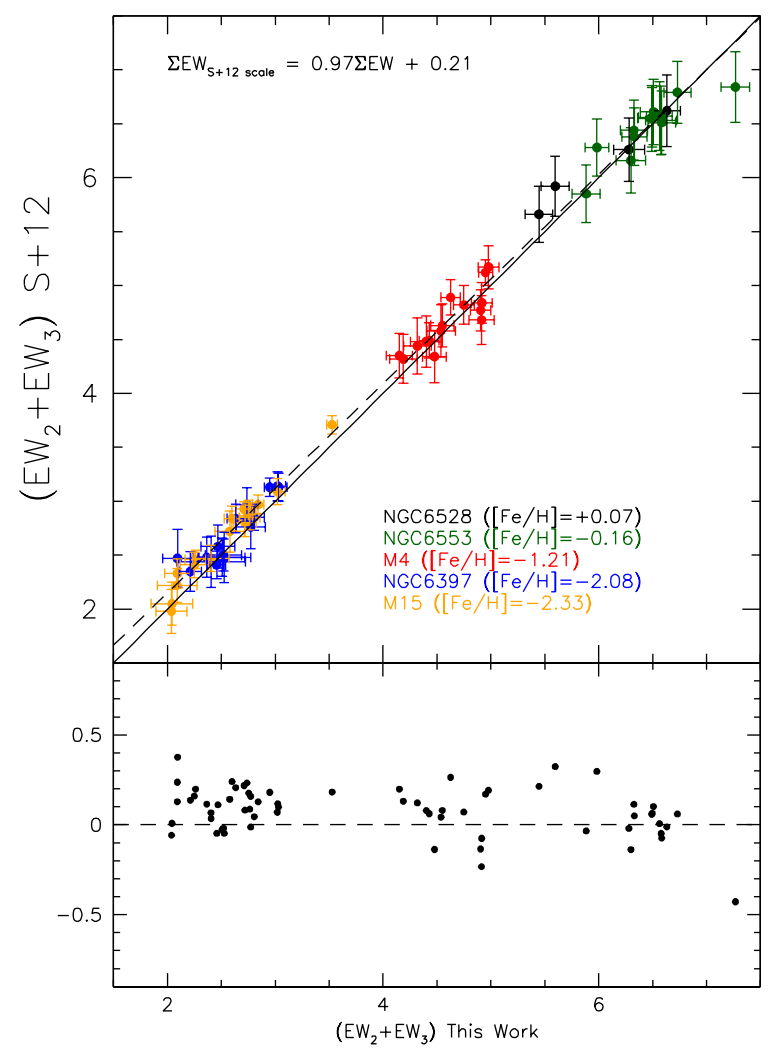

Fig. 5. Sum of EWs of the two strongest CaT lines for stars in 5 template globular clusters measured by Saviane et al. (2012) is plotted against our own measurements. The error bars were computed as in Vásquez et al. (2015). The one-to-one relation is shown as a black continuous line, whereas the dashed line is a linear fit to the data. The residuals from the one-to-one line are shown in the lower panel.

where $V$ and $V_{\mathrm{HB}}$ are the visual magnitude of each RGB star and the mean magnitude of the $\mathrm{HB}$, respectively. The $V$ magnitude difference with respect to the $\mathrm{HB}$ is not affected by errors in distance, reddening, or photometric zero-point, while it is affected by internal differential reddening. In a plot of $\Sigma_{\mathrm{EW}}$ vs. $\left(V-V_{\mathrm{HB}}\right)$, all measurements in a globular cluster follow a linear relation for member stars brighter than the HB (Fig. 6). The slope is approximately constant in the metallicity range of globular clusters $(-2.5<[\mathrm{Fe} / \mathrm{H}]<+0.0)$, although there is evidence that this assumption loses validity outside this range, especially for extremely metal poor stars (Starkenburg et al. 2010; Carrera et al. 2013). The average slope calculated using our EW measurements is $\beta=-0.55$, only slightly different from the value found by Saviane et al. (2012).

By removing the dependence on gravity and $T_{\mathrm{eff}}, W^{\prime}$ can be used to determine the metallicity of a given RGB star by using a suitable calibration.

Stellar magnitudes and the HB level have been measured using point spread function (PSF) photometry on the preimaging data, using Stetson's DAOPHOT/ALLSTAR package (Stetson 1987, 1994). All the instrumental magnitudes were calibrated using colour terms and zero points provided by ESO as part of their routine quality control ${ }^{2}$. As not all of the pre-imaging observations were taken in photometric conditions, the final magnitudes are only approximately on the $V, I$ standard system.

\footnotetext{
2 See http://www.eso.org/observing/dfo/quality/FORS2/ qc/photcoeff/photcoeffs_fors2.html
}

However, this is not relevant to the metallicity calibration due to the differential dependence on $V-V_{\mathrm{HB}}$.

To determine the HB level for each cluster, we used the CMD of NGC 6864 to define a template HB. This cluster has a well defined blue $\mathrm{HB}$ along with a red $\mathrm{HB}$, which makes the construction of a fiducial line easier. As a first approximation we chose the fiducial line published by Catelan et al. (2002), shifted in colour and magnitude to match the observed CMD (see right panel of Fig. 7). This HB template was used to match the CMDs using stars within the cluster half-light radius (given in the Harris catalogue). Examples are shown in Fig. 7. This selection minimises the field star contamination and makes it easier to recognise the HB morphology. As the HB morphology changes with cluster metallicity, being blue and extended for metal poor clusters and limited to a red HB for metal rich clusters, the matching criteria as well as the error on the HB level differ from case to case. For clusters with a red $\mathrm{HB}$, the mean and dispersion of red $\mathrm{HB}$ stars define $V_{\mathrm{HB}}$ and its error $(1 \sigma)$. For metal poor clusters the mean level and its uncertainty ( $1 \sigma$ around the line) are defined by the stars close to the horizontal portion of the fiducial line. The measurement of $V_{\mathrm{HB}}$ is also affected by differential reddening, which is difficult to correct without a proper reddening map for the cluster. To take into account the uncertainty introduced by this effect, no selection was applied to the stars used to measure $V_{\mathrm{HB}}$. The variable reddening increases the $1 \sigma$ error on $V_{\mathrm{HB}}$. The most uncertain value was derived for Terzan 1 and Terzan 2 with $\Delta V_{\mathrm{HB}} \sim 0.35 \mathrm{mag}$, which leads to an uncertainty of $\sim 0.15$ dex in metallicity. For the rest of the sample the errors on $V_{\mathrm{HB}}$ are typically $\sim 0.1 \mathrm{mag}$. The $V-V_{\mathrm{HB}}$ values of member stars are given in Table 3 .

\subsection{Metallicity calibration}

In Saviane et al. (2012) the reduced equivalent widths were converted into $[\mathrm{Fe} / \mathrm{H}]$ values using the empirical calibration

$[\mathrm{Fe} / \mathrm{H}]_{\mathrm{S} 12}=0.0178 W^{\prime 3}-0.114 W^{\prime 2}+0.599 W^{\prime}-3.113$,

based on a set of template globular clusters with well-established abundances from high resolution spectroscopy. This relation has an r.m.s. dispersion around the fit of 0.13 dex and is defined in the $[\mathrm{Fe} / \mathrm{H}]$ range from -2.33 dex to +0.07 dex. A cubic relation between $W^{\prime}$ and $[\mathrm{Fe} / \mathrm{H}]$ was needed to fit the most metal-rich and metal-poor globular clusters in the calibration sample. A slight curvature in the CaT calibration appears to be common to several published calibrations based on globular clusters (e.g., Rutledge et al. 1997; Carretta et al. 2009; Carrera et al. 2013). As long as the calibration templates and the target clusters share the same chemical evolution history and are measured in the same way (as in our case), the shape of the empirical calibration is not critical and there is no theoretical reason for it to be linear. The main caveat is represented here by the paucity of globular cluster calibrators at high metallicity: indeed, Eq. (2) was based on just three GCs more metal-rich that $[\mathrm{Fe} / \mathrm{H}]=-0.7$.

On the other hand, linear calibrations have been obtained by extending the fit using metal-rich stars chosen outside the globular cluster population (e.g., Cole et al. 2004; Carrera et al. 2013, and references therein). The most recent examples are those of Vásquez et al. (2015) and Da Costa (2016). Vásquez et al. (2015) proposed a new metallicity calibration on the C09 scale, using red giant and red clump stars in the Galactic bulge to define the metal-rich end of the calibration, and globular clusters giants from Warren \& Cole (2009) at the metal-poor end. Each sample was analysed independently, finding linear calibrations between $W^{\prime}$ and metallicity with a small difference in slope $(<7 \%)$. To 
Table 3. Programme cluster data.

\begin{tabular}{|c|c|c|c|c|c|c|c|c|c|c|}
\hline Name & Slit & RA (2000) & Dec (2000) & $\begin{array}{l}\mathrm{RV} \\
(\mathrm{km} \mathrm{s}\end{array}$ & eRV & $\begin{array}{c}\Delta V \\
(\mathrm{mag})\end{array}$ & $\begin{array}{c}\Sigma_{\mathrm{EW}}(\mathrm{c}) \\
(\AA)\end{array}$ & $\mathrm{e} \Sigma_{\mathrm{EW}}(\mathrm{c})$ & $\begin{array}{l}\Sigma_{\text {EW }}(\mathrm{m}) \\
(\AA)\end{array}$ & $\mathrm{e} \Sigma_{\mathrm{EW}}(\mathrm{m})$ \\
\hline BH 176 & 1_668 & $: 12.4$ & $-50: 2: 18.9$ & -2.30 & 1.53 & -0.73 & 6.93 & 0.53 & 6.92 & 0.22 \\
\hline BH 176 & 1_1578 & $15: 39: 12.6$ & $-50: 1: 59.0$ & -5.42 & 1. & -0.82 & 6.30 & 0.39 & 6.28 & 0.16 \\
\hline BH 176 & $1 \_2513$ & $15: 39: 10.4$ & $-50: 1: 38.6$ & 4.63 & 3.62 & +0.18 & 6.12 & 0.65 & 6.09 & 0.28 \\
\hline BH 176 & $1 \_3686$ & $15: 38: 55.8$ & $-50: 1: 09.8$ & 7.31 & 1.49 & -1.03 & 7.63 & 0.46 & 7.65 & 0.17 \\
\hline BH 176 & 1_4579 & $15: 39: 07.0$ & $-50: 0: 47.3$ & 4.97 & 1.78 & -0.39 & 6.21 & 0.49 & 6.19 & 0.21 \\
\hline
\end{tabular}

Notes. This table is available in its entirety in a machine-readable form at the CDS. A few lines are shown here to illustrate the table content.

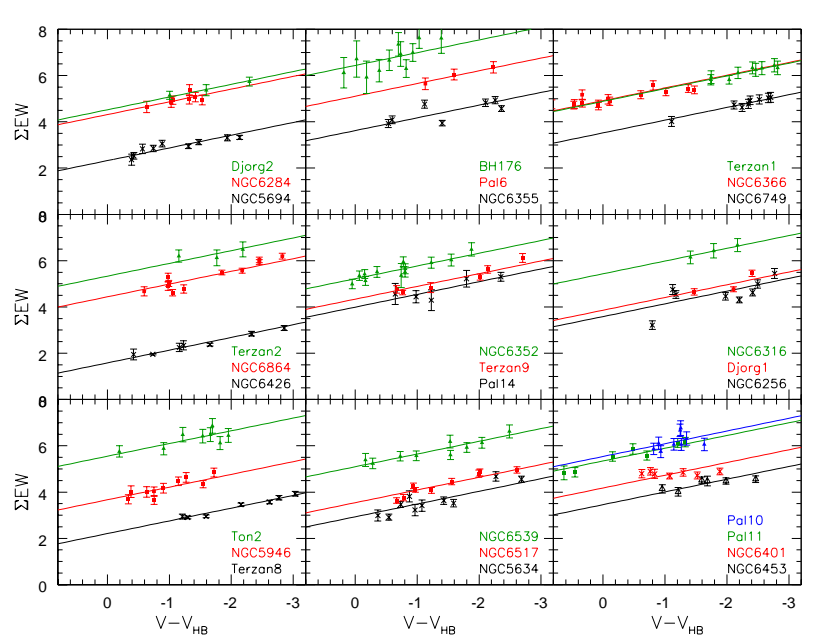

Fig. 6. Sum of the EWs of the two strongest CaT lines, $\Sigma_{\mathrm{EW}}$, is plotted against the magnitude difference from the horizontal branch $\left(V-V_{\mathrm{HB}}\right)$ for the 28 programme clusters. Different colours are used to identify stars belonging to different clusters, together with best fitting lines for the present data with a common fixed slope $\beta=-0.55$.

take into account this small difference, a quadratic polynomial was used to link the fits to the bulge stars and GCs:

$[\mathrm{Fe} / \mathrm{H}]_{\mathrm{V} 15}=0.006 W^{\prime 2}+0.432 W^{\prime}-3.150$,

with an r.m.s. spread of 0.197 dex. The curvature of this relation is very modest so it can be regarded as nearly linear. Da Costa (2016) used a combination of Galactic globular clusters and open clusters to establish a linear relation between $W^{\prime}$ and $[\mathrm{Fe} / \mathrm{H}]$ in the metallicity range $-2.4<[\mathrm{Fe} / \mathrm{H}]<+0.1$. The fit is:

$[\mathrm{Fe} / \mathrm{H}]_{\mathrm{DC} 16}=(0.528 \pm 0.017) W^{\prime}-(3.420 \pm 0.077)$,

with an r.m.s. of the residuals of $0.06 \mathrm{dex}$. This relation is consistent with that of Saviane et al. (2012) up to the metallicity of 47 Tuc.

All these $[\mathrm{Fe} / \mathrm{H}]-\left\langle W^{\prime}\right\rangle$ calibrations are shown in Fig. 8 (left panel), along with the Rutledge et al. (1997) calibration corrected to the $W^{\prime}$ definition of Saviane et al. (2012). The relations are consistent for metallicities up to $[\mathrm{Fe} / \mathrm{H}] \sim-0.7$, while they diverge for metal-rich clusters. In Fig. 8 they are overplotted on a sub-set of our catalogue in common with Dias et al. $(2015,2016 a)^{3}$. In the parallel work of Dias et al., metallicities were obtained from spectral fitting of medium-resolution spectra in the interval $4560-5860 \AA$, along with estimates of

\footnotetext{
348 of our 56 clusters have been observed in the framework of our GGC survey using the same slit masks in the optical and near-infrared wavelength ranges.
}

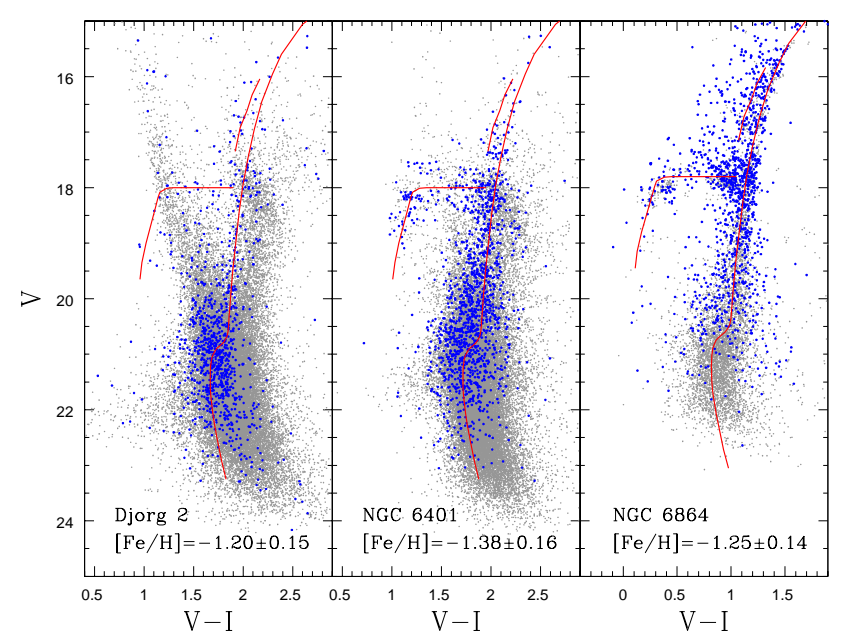

Fig. 7. Optical CMDs of Djorg 2, NGC 6401 and NGC 6864. The full photometric catalogues (cluster + field) obtained from our FORS2 preimaging are plotted in grey, while blue dots are used for the inner regions $(r<0.5 \operatorname{arcmin})$. The fiducial loci published by Catelan et al. (2002) for NGC 6864 are over-plotted as a red curve.

the stellar parameters and alpha-element abundances. Since the $[\mathrm{Fe} / \mathrm{H}]$ values of Dias et al. (2016a) are in excellent agreement with high-resolution spectroscopic metallicities in the range $-2.5<[\mathrm{Fe} / \mathrm{H}]<0.0$ (see discussion in D16), they provide an independent $\mathrm{CaT}$ calibration:

$[\mathrm{Fe} / \mathrm{H}]_{\mathrm{D} 16}=(0.055 \pm 0.012) W^{\prime 2}+(0.13 \pm 0.10) W^{\prime}-(2.68 \pm 0.17)$

with r.m.s. $=0.11$ dex and coefficient of determination $r^{2}=0.97$. The new relation is shown in Fig. 8 (left panel) as a continuous line. The fit appears to confirm the S12 calibration in the validity range for globular clusters. However, it is now based on 17 clusters more metal-rich than $[\mathrm{Fe} / \mathrm{H}]=-0.7$, giving higher weight to the non-linear behaviour found for globular clusters by S12 and other authors.

The right panels of Fig. 8 show the residuals between the $[\mathrm{Fe} / \mathrm{H}]$ values computed using different $\mathrm{CaT}$ calibrations and the metallicities in D16 for all clusters in common. The CaT metallicities are the average of individual metallicities computed for member stars. The error bars represent the uncertainties in our determinations which are a combination of the statistical uncertainty in the EWs, the calibration uncertainty (taken as the rms dispersion about the fit) and, where necessary, inclusion of allowance for significant differential reddening that can affect the individual $V_{\mathrm{HB}}$ values (see S12 for details). It is apparent from Fig. 8 that non-linear calibrations provide a better fit for globular clusters, with the exception of the metal-rich cluster BH 176 which 
S. Vásquez et al.: Homogeneous metallicities and radial velocities for Galactic globular clusters. II.
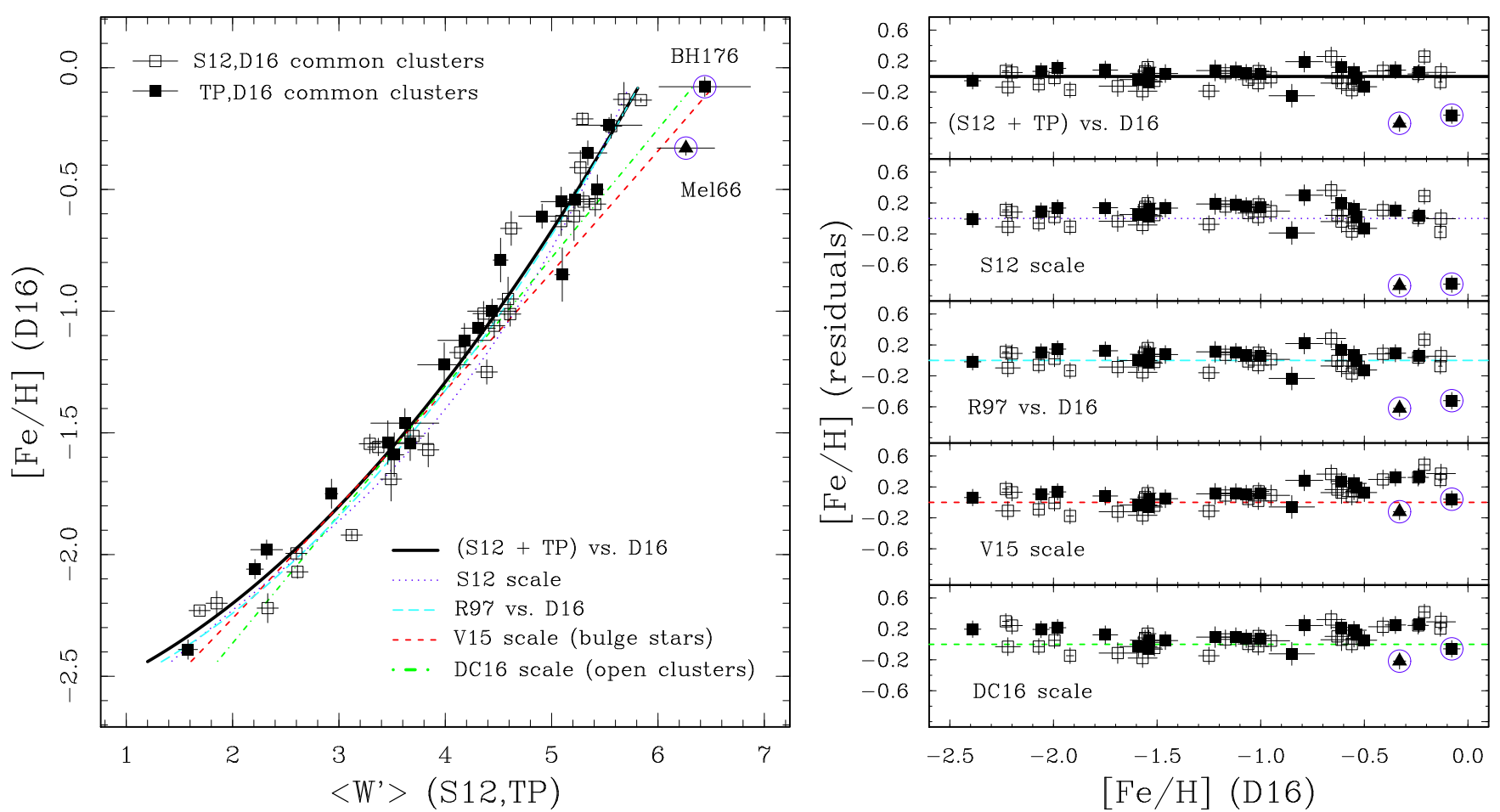

Fig. 8. Comparison between the metallicities of GGCs derived from the optical spectral range (Dias et al. 2016a) and the mean reduced equivalent widths $\left\langle W^{\prime}\right\rangle$ for the clusters in common with data in this paper (filled squares) and the S12 sample (open squares). The peculiar cluster BH 176 and the open cluster Mel 66 are marked by open circles. Different CaT calibrations are shown: the best fit to the merged FORS2 sample as a black solid line, the Saviane et al. (2012) relation as a blue dotted line, and the Rutledge et al. (1997) calibration (converted to our $W^{\prime}$ scale) as a cyan dashed line. These relations based on globular cluster data share a common curved trend. In addition, the Vásquez et al. (2015) fit which includes bulge stars on the metal-rich side is shown as a red dashed line, and the calibration of Da Costa (2016) based on stars in globular and open clusters is represented by the green dot-dashed line. In the right panels we plot the $[\mathrm{Fe} / \mathrm{H}]$ residuals (this work - D16) for five CaT calibrations. See discussion in the text.

has anomalously large $W^{\prime}$. The position of $\mathrm{BH} 176$ in the $[\mathrm{Fe} / \mathrm{H}]$ vs. $\left\langle W^{\prime}\right\rangle$ plot is more similar to the open cluster Melotte 66 than to other metal-rich GCs. The $\left\langle W^{\prime}\right\rangle$ of Melotte 66 was remeasured with our code using spectra from Da Costa (2016). Our $\left\langle W^{\prime}\right\rangle$ value given in Fig. 8, although larger than the original measurement of Da Costa (2016) lying pretty much exactly on the author's calibration line, confirms that the $W^{\prime}$ values measured for stars in this open cluster (as well as in BH 176) are larger than those obtained in GCs of similar metallicity. At present, a full understanding of the calibration of the CaT line strengths for metal-rich stars calls for further investigation and theoretical modelling of behaviour of these lines in giant stars as a function of the stellar parameters $\log g, T_{\text {eff }},[\mathrm{Fe} / \mathrm{H}]$, and $[\alpha / \mathrm{Fe}]$ or, equivalently, the population properties age and metallicity.

The metallicities of the 28 globular clusters measured in this paper are therefore listed in Table 4 using different calibrations, although we maintain the S12 metallicity scale as our reference calibration. The reduced EWs listed in these tables are given on the measurement system of Saviane et al. (2012) and the metallicities are calculated on the scales of Dias et al. (2016a, Eq. (5)), Saviane et al. (2012, Eq. (2)), and Vásquez et al. (2015, Eq. (3)). The errors are computed as in S12 and Fig. 8. We note that, in some cases, the metallicities are based on a few member stars only and should be regarded as more dubious than suggested by the internal error. In the case of the V15 calibration the errors are dominated by a large calibration uncertainty of 0.197 dex. Further, the listed $W^{\prime}$ can be used to calculate the metallicities on the scale of Da Costa (2016, Eq. (4)). For the sake of completeness, the clusters in Saviane et al. (2012) are listed in Table 5 using the same calibrations. The cluster metallicities from the compilations of Dias et al. (2016b), Harris (1996) and C09 are also given for comparison.

\section{Individual cluster results}

Some clusters present special characteristics in terms of possible metallicity spread, differential reddening effects and differences in radial velocity and $[\mathrm{Fe} / \mathrm{H}]$ with respect to the literature. As discussed in Saviane et al. (2012), we calculated the ratio $\rho$ between the r.m.s. dispersion around the linear fit with fixed slope (here $\beta=-0.55 \AA \mathrm{mag}^{-1}$ ) and the average measurement error in $\Sigma_{\mathrm{EW}}$. This indicator of possible abundance spreads is given in the last column in Tables 4 and 5. Clusters with $\rho \geq 2$ are considered candidates for a metallicity dispersion, while clusters with $1<\rho<2$ are marginal candidates.

\section{1. $\mathrm{BH} 176$}

The metal rich cluster BH 176 (ESO 224-SC8) is regarded as a transition stellar system, classified as either an old metalrich open cluster or a young metal-rich globular cluster with unclear origin (Ortolani et al. 1995a; Phelps \& Schick 2003). From isochrone fitting, Frinchaboy et al. (2006) found that the CMD is consistent with solar metallicity $([\mathrm{Fe} / \mathrm{H}]=0.0$ to $[\mathrm{Fe} / \mathrm{H}]=+0.2$ ), with an age range from 5.6 to $7.1 \mathrm{Gyr}$. In a more recent analysis Davoust et al. (2011) found a metallicity $[\mathrm{Fe} / \mathrm{H}]=-0.1 \pm 0.1$ and age $\sim 7 \mathrm{Gyr}$ by combining near-infrared 2MASS and optical photometry, a result confirmed by mediumresolution stellar spectroscopy by Sharina et al. (2014). 
A\&A 619, A13 (2018)

Table 4. Metallicities of programme clusters using the D16, S12, and V15 calibrations, compared with $[\mathrm{Fe} / \mathrm{H}]$ from the literature.

\begin{tabular}{|c|c|c|c|c|c|c|c|c|c|c|}
\hline Cluster & $N$ & $\left\langle W^{\prime}\right\rangle$ & $\begin{array}{c}{[\mathrm{Fe} / \mathrm{H}]_{\mathrm{CaT}}} \\
\text { D16 cal. }\end{array}$ & $\begin{array}{c}{[\mathrm{Fe} / \mathrm{H}]_{\mathrm{CaT}}} \\
\text { S12 cal. }\end{array}$ & $\begin{array}{c}{[\mathrm{Fe} / \mathrm{H}]_{\mathrm{CaT}}} \\
\mathrm{V} 15 \mathrm{cal} .\end{array}$ & {$[\mathrm{Fe} / \mathrm{H}]_{\mathrm{D} 16}$} & {$[\mathrm{Fe} / \mathrm{H}]_{\mathrm{H} 10}$} & $w$ & {$[\mathrm{Fe} / \mathrm{H}]_{\mathrm{C} 09}$} & $\rho$ \\
\hline BH 176 & 11 & $6.44 \pm 0.43$ & & & $-0.12 \pm 0.22$ & $-0.08 \pm 0.04$ & 0.00 & 1 & & 0.7 \\
\hline Djorg 1 & 3 & $3.86 \pm 0.26$ & $-1.36 \pm 0.14$ & $-1.48 \pm 0.15$ & $-1.39 \pm 0.21$ & $-1.54 \pm 0.13^{a}$ & -1.51 & 1 & $\ldots$ & 1.5 \\
\hline Djorg 2 & 3 & $4.52 \pm 0.07$ & $-0.97 \pm 0.13$ & $-1.09 \pm 0.15$ & $-1.07 \pm 0.21$ & $-0.79 \pm 0.09$ & -0.65 & 1 & & 0.4 \\
\hline NGC 5634 & 10 & $2.93 \pm 0.28$ & $-1.83 \pm 0.12$ & $-1.89 \pm 0.13$ & $-1.83 \pm 0.20$ & $-1.75 \pm 0.06$ & -1.88 & 2 & $-1.93 \pm 0.09$ & 1.3 \\
\hline NGC 5694 & 9 & $2.32 \pm 0.15$ & $-2.08 \pm 0.11$ & $-2.11 \pm 0.13$ & $-2.12 \pm 0.20$ & $-1.98 \pm 0.06$ & -1.98 & 5 & $-2.02 \pm 0.07$ & 1.0 \\
\hline NGC 5946 & 10 & $3.67 \pm 0.22$ & $-1.46 \pm 0.13$ & $-1.57 \pm 0.14$ & $-1.48 \pm 0.21$ & $-1.54 \pm 0.07$ & -1.29 & 4 & $-1.29 \pm 0.14$ & 0.9 \\
\hline NGC 6256 & 10 & $3.58 \pm 0.44$ & $-1.51 \pm 0.14$ & $-1.61 \pm 0.14$ & $-1.53 \pm 0.21$ & $-1.05 \pm 0.13^{a}$ & -1.02 & 2 & $-0.62 \pm 0.09$ & 2.2 \\
\hline NGC 6284 & 7 & $4.31 \pm 0.16$ & $-1.10 \pm 0.12$ & $-1.22 \pm 0.14$ & $-1.18 \pm 0.20$ & $-1.07 \pm 0.06$ & -1.26 & 2 & $-1.31 \pm 0.09$ & 0.7 \\
\hline NGC 6316 & 3 & $5.43 \pm 0.06$ & $-0.35 \pm 0.14$ & $-0.37 \pm 0.17$ & $-0.63 \pm 0.21$ & $-0.50 \pm$ & 5 & - & $-0.36 \pm 0.14$ & 0.1 \\
\hline NGC 6352 & 12 & $5.22 \pm 0.18$ & $-0.50 \pm 0.12$ & $-0.56 \pm 0.14$ & $-0.73 \pm 0.20$ & $-0.54 \pm 0.04$ & -0.64 & $J$ & $-0.62 \pm 0.05$ & 0.7 \\
\hline NGC 6355 & 7 & $3.62 \pm 0.32$ & $-1.49 \pm 0.14$ & $-1.59 \pm 0.15$ & $-1.51 \pm 0.21$ & $-1.46 \pm 0.06$ & -1.37 & 4 & $-1.33 \pm 0.14$ & 1.6 \\
\hline NGC 6366 & 14 & $4.91 \pm 0.22$ & $-0.72 \pm 0.12$ & $-0.81 \pm 0.14$ & $-0.88 \pm 0.20$ & $-0.61 \pm 0.05$ & -0.59 & 3 & $-0.59 \pm 0.08$ & 1.3 \\
\hline NGC 6401 & 7 & $4.18 \pm 0.25$ & $-1.18 \pm 0.14$ & $-1.30 \pm 0.15$ & $-1.24 \pm 0.21$ & $-1.12 \pm 0.07$ & -1.02 & 5 & $-1.01 \pm 0.14$ & 1.3 \\
\hline NGC 6426 & 7 & $1.58 \pm 0.09$ & $-2.34 \pm 0.11$ & $-2.38 \pm 0.13$ & $-2.45 \pm 0.20$ & $-2.39 \pm 0.04$ & -2.15 & 3 & & 0.8 \\
\hline NGC 6453 & 6 & $3.46 \pm 0.17$ & $-1.57 \pm 0.12$ & $-1.67 \pm 0.13$ & $-1.58 \pm 0.20$ & $-1.54 \pm 0.09$ & -1.50 & 4 & $-1.48 \pm 0.14$ & 1.0 \\
\hline NGC 6517 & 9 & $3.54 \pm 0.18$ & $-1.53 \pm 0.12$ & $-1.63 \pm 0.13$ & $-1.55 \pm 0.20$ & $-1.26 \pm 0.13^{a}$ & -1.23 & 4 & $-1.24 \pm 0.14$ & 1.1 \\
\hline NGC 6539 & 9 & $5.09 \pm 0.19$ & $-0.59 \pm 0.12$ & $-0.67 \pm 0.14$ & $-0.80 \pm 0.20$ & $-0.55 \pm 0.06$ & -0.63 & 5 & $-0.53 \pm 0.14$ & 0.8 \\
\hline NGC 6749 & 8 & $3.52 \pm 0.09$ & $-1.54 \pm 0.11$ & $-1.64 \pm 0.13$ & $-1.56 \pm 0.20$ & $-1.59 \pm 0.09$ & -1.60 & 1 & $-1.62 \pm 0.09$ & 0.3 \\
\hline NGC 6864 & 11 & $4.44 \pm 0.23$ & $-1.02 \pm 0.12$ & $-1.14 \pm 0.14$ & $-1.11 \pm 0.20$ & $-1.00 \pm 0.05$ & -1.29 & 3 & $-1.29 \pm 0.14$ & 1.3 \\
\hline Pal 10 & 9 & $5.54 \pm 0.31$ & $-0.27 \pm 0.16$ & $-0.27 \pm 0.19$ & $-0.57 \pm 0.21$ & $-0.24 \pm 0.03$ & -0.10 & 1 & & 1.0 \\
\hline Pal 11 & 7 & $5.34 \pm 0.18$ & $-0.42 \pm 0.13$ & $-0.45 \pm 0.15$ & $-0.67 \pm 0.20$ & $-0.35 \pm 0.05$ & -0.40 & 4 & $-0.45 \pm 0.08$ & 0.7 \\
\hline Pal 14 & 5 & $3.99 \pm 0.25$ & $-1.29 \pm 0.13$ & $-1.41 \pm 0.14$ & $-1.33 \pm 0.21$ & $-1.22 \pm 0.09$ & -1 & 4 & $-1.63 \pm 0.08$ & 0.7 \\
\hline Pal 6 & 3 & & & & & & & 3 & $-1.06 \pm 0.09$ & 0.2 \\
\hline Terzan 1 & 9 & $4.88 \pm 0.10$ & $-0.74 \pm 0.18$ & $-0.84 \pm 0.21$ & $-0.90 \pm 0.23$ & $-1.06 \pm 0.13^{a}$ & -1.03 & 3 & $-1.29 \pm 0.09$ & 0.3 \\
\hline Terzan 2 & 3 & $5.33 \pm 0.21$ & $-0.42 \pm 0.18$ & $-0.46 \pm 0.22$ & $-0.68 \pm 0.22$ & $-0.72 \pm 0.13^{a}$ & -0.69 & 3 & $-0.29 \pm 0.09$ & 0.6 \\
\hline Terzan 8 & 7 & $2.21 \pm 0.08$ & $-2.12 \pm 0.11$ & $-2.15 \pm 0.13$ & $-2.17 \pm 0.20$ & $-2.06 \pm 0.04$ & -2.16 & 2 & & 0.8 \\
\hline Terzan 9 & 6 & $4.34 \pm 0.19$ & $-1.08 \pm 0.14$ & $-1.21 \pm 0.15$ & $-1.16 \pm 0.21$ & $-1.08 \pm 0.13^{a}$ & -1.05 & 2 & $-2.07 \pm 0.09$ & 0.7 \\
\hline Ton 2 & 8 & $5.55 \pm 0.26$ & $-0.26 \pm 0.15$ & $-0.26 \pm 0.19$ & $-0.57 \pm 0.21$ & $-0.73 \pm 0.13^{a}$ & -0.70 & 1 & $\ldots$ & 0.8 \\
\hline
\end{tabular}

Notes. Columns: (1) Cluster ID. (2) Number of cluster member stars analysed. (3) Average reduced EW of clusters in our sample on the measurement scale of Saviane et al. (2012), as discussed in Sect. 4.1. (4) Metallicity calibrated on the D16 scale using Eq. (5), and total uncertainty. $(5,6)$ The same for the S12 and V15 scales, using Eqs. (2) and (3). (7) Average metallicity from Dias et al. (2016a) if present, or the homogeneous compilation by Dias et al. (2016b) on the metallicity scale of Dias et al. (2016a). (8,9) Metallicity and weight from the Harris catalogue. (10) Average metallicity from literature compiled by Carretta et al. (2009). (11) Ratio of the rms dispersion around the linear fit with fixed slope, to the mean measurement error. ${ }^{(a)}$ Metallicities from Dias et al. (2016b).

As shown in Fig. 6 and Table 4, after applying our selection criteria 11 stars were selected as members, with a relatively large dispersion in $\left\langle W^{\prime}\right\rangle(0.43 \AA)$ and a narrow radial velocity distribution $\left(\sigma_{v_{\mathrm{r}}} \approx 5 \mathrm{~km} \mathrm{~s}^{-1}\right)$ with average zero. This value agrees with previous measurements, in particular that of Sharina et al. (2014), and does not overlap the radial velocity of field stars expected from the Besançon model (Robin et al. 2003), which is $v_{\mathrm{r}}=-71 \mathrm{~km} \mathrm{~s}^{-1}$ with a dispersion of $50 \mathrm{~km} \mathrm{~s}^{-1}$. We also tested the contamination of the sample by foreground dwarf stars using the $\lambda 8806.8 \AA \mathrm{Mg}$ line. As shown by Battaglia \& Starkenburg (2012), the EW ratio between the $\mathrm{Mg}$ line and the two strongest $\mathrm{CaT}$ lines can be used to discriminate between dwarf and giant stars, due to the $\mathrm{Mg}$ line dependence on gravity. This method is particularly useful when the cluster and foreground samples have different metallicities (see Fig. 7 in Da Costa et al. 2014). However, BH 176 looks as metal rich as the Milky Way disk, which makes this comparison rather difficult. From this method we identified two foreground dwarf stars. Excluding them does not change the mean metallicity of the cluster.

From our set of member stars we measured $\left\langle W^{\prime}\right\rangle=6.44 \AA$, which is the highest reduced equivalent width found in our sample, and suggests a high metallicity in agreement with the photometric results. Applying the calibration of Saviane et al. (2012) or Eq. (5) would require a large extrapolation (these relations are defined in the range $\sim 1.8<W^{\prime}<5.8$ ) and give an unrealistic iron abundance $[\mathrm{Fe} / \mathrm{H}] \geqslant+0.4$. The less steep calibration of Vásquez et al. (2015) yields a value for the BH 176 mean metallicity $[\mathrm{Fe} / \mathrm{H}]=-0.12 \pm 0.22$, in excellent agreement with the results of full-spectrum fitting of optical spectra by Sharina et al. (2014) and Dias et al. (2016a) $([\mathrm{Fe} / \mathrm{H}]=-0.08 \pm 0.04)$. Its location in Fig. 8, where it appears to be stronger lined than GCs of similar metallicity, suggests that BH 176 is an old metal-rich open cluster rather than a young metal-rich GC.

\subsection{Djorgovski 1}

This bulge cluster is one of the two faintest objects in our sample (together with Terzan 1), with a mean $\mathrm{HB}$ magnitude $V_{\mathrm{HB}}=$ 21.15. It is located at $(l, b)=(-3.33,-2.48)$, in a high-extinction area in the direction of the Galactic centre. NTT V,I CMDs of Djorg 1 by Ortolani et al. (1995b) gave a distance of $8.8 \mathrm{kpc}$ and high reddening $E(B-V)=1.71$. The CMD was very dispersed, despite the good observing seeing. Valenti et al. (2010), by cleaning the CMD for contamination by the MW foreground, found a lower reddening and larger distance $([E(B-V)=1.58]$, $13.7 \mathrm{kpc})$, and a metal-poor composition $([\mathrm{Fe} / \mathrm{H}]=-1.51)$.

In our study, the target selection from the CMD was rather difficult, and indeed only three stars have been classified as cluster 
S. Vásquez et al.: Homogeneous metallicities and radial velocities for Galactic globular clusters. II.

Table 5. Metallicities of globular clusters in Saviane et al. (2012).

\begin{tabular}{|c|c|c|c|c|c|c|c|c|c|c|}
\hline Cluster & $N$ & $\left\langle W^{\prime}\right\rangle$ & $\begin{array}{c}{[\mathrm{Fe} / \mathrm{H}]_{\mathrm{CaT}}} \\
\mathrm{D} 16 \mathrm{cal} .\end{array}$ & $\begin{array}{c}{[\mathrm{Fe} / \mathrm{H}]_{\mathrm{CaT}}} \\
\mathrm{S} 12 \mathrm{cal} .\end{array}$ & $\begin{array}{c}{[\mathrm{Fe} / \mathrm{H}]_{\mathrm{CaT}}} \\
\mathrm{V} 15 \mathrm{cal} .\end{array}$ & {$[\mathrm{Fe} / \mathrm{H}]_{\mathrm{D} 16}$} & {$[\mathrm{Fe} / \mathrm{H}]_{\mathrm{H} 10}$} & $w$ & {$[\mathrm{Fe} / \mathrm{H}]_{\mathrm{C} 09}$} & $\rho$ \\
\hline Pyxis & 5 & $3.90 \pm 0.24$ & $-1.34 \pm 0.13$ & $-1.45 \pm 0.14$ & $-1.37 \pm 0.20$ & $-1.40 \pm 0.13^{a}$ & -1.20 & 3 & & 0.8 \\
\hline NGC 2808 & 17 & $4.46 \pm 0.20$ & $-1.01 \pm 0.11$ & $-1.13 \pm 0.13$ & $-1.10 \pm 0.20$ & $-1.06 \pm 0.05$ & -1.14 & 4 & $-1.18 \pm 0.04$ & 1.0 \\
\hline Rup 106 & 9 & $3.29 \pm 0.25$ & $-1.66 \pm 0.12$ & $-1.74 \pm 0.13$ & $-1.66 \pm 0.20$ & $-1.54 \pm 0.04$ & -1.68 & 6 & $-1.78 \pm 0.08$ & 1.9 \\
\hline NGC 5824 & 17 & $2.60 \pm 0.30$ & $-1.97 \pm 0.11$ & $-2.01 \pm 0.13$ & $-1.99 \pm 0.20$ & $-1.99 \pm 0.03$ & -1.91 & 4 & $-1.94 \pm 0.14$ & 2.2 \\
\hline Lynga 7 & 8 & $5.21 \pm 0.11$ & $-0.51 \pm 0.13$ & $-0.57 \pm 0.15$ & $-0.74 \pm 0.20$ & $-0.61 \pm 0.10$ & -1.01 & 3 & & 0.3 \\
\hline NGC 6139 & 15 & $3.54 \pm 0.15$ & $-1.53 \pm 0.11$ & $-1.63 \pm 0.13$ & $-1.55 \pm 0.20$ & $-1.58 \pm 0.07^{a}$ & -1.65 & 2 & $-1.71 \pm 0.09$ & 1.0 \\
\hline Terzan 3 & 10 & $4.54 \pm 0.12$ & $-0.96 \pm 0.13$ & $-1.08 \pm 0.14$ & $-1.07 \pm 0.20$ & $-1.01 \pm 0.08^{a}$ & -0.74 & 2 & & 0.4 \\
\hline NGC 6325 & 10 & $3.99 \pm 0.20$ & $-1.29 \pm 0.12$ & $-1.41 \pm 0.13$ & $-1.33 \pm 0.20$ & $-1.38 \pm 0.10^{a}$ & -1.25 & 4 & $-1.37 \pm 0.14$ & 1.2 \\
\hline NGC 6356 & 11 & $5.30 \pm 0.15$ & $-0.45 \pm 0.11$ & $-0.49 \pm 0.13$ & $-0.69 \pm 0.20$ & $-0.55 \pm 0.04$ & -0.40 & 5 & $-0.35 \pm 0.14$ & 0.5 \\
\hline HP 1 & 8 & $4.14 \pm 0.30$ & $-1.20 \pm 0.13$ & $-1.32 \pm 0.14$ & $-1.26 \pm 0.20$ & $-1.17 \pm 0.07$ & -1.00 & 4 & $-1.57 \pm 0.09$ & 1.3 \\
\hline NGC 6380 & 8 & $5.31 \pm 0.18$ & $-0.44 \pm 0.14$ & $-0.48 \pm 0.16$ & $-0.69 \pm 0.20$ & $-0.47 \pm 0.14^{a}$ & -0.75 & 2 & $-0.40 \pm 0.09$ & 0.7 \\
\hline NGC 6440 & 8 & $5.56 \pm 0.18$ & $-0.26 \pm 0.13$ & $-0.25 \pm 0.16$ & $-0.56 \pm 0.20$ & $-0.24 \pm 0.05$ & -0.36 & 6 & $-0.20 \pm 0.14$ & 0.6 \\
\hline NGC 6441 & 7 & $5.27 \pm 0.17$ & $-0.47 \pm 0.14$ & $-0.52 \pm 0.16$ & $-0.71 \pm 0.20$ & $-0.41 \pm 0.07$ & -0.46 & 6 & $-0.44 \pm 0.07$ & 0.6 \\
\hline NGC 6558 & 4 & $4.61 \pm 0.15$ & $-0.91 \pm 0.12$ & $-1.03 \pm 0.14$ & $-1.03 \pm 0.20$ & $-1.01 \pm 0.05$ & -1.32 & 5 & $-1.37 \pm 0.14$ & 0.9 \\
\hline Pal 7 & 14 & $5.41 \pm 0.12$ & $-0.37 \pm 0.12$ & $-0.39 \pm 0.14$ & $-0.64 \pm 0.20$ & $-0.56 \pm 0.05$ & -0.75 & 1 & $-0.65 \pm 0.09$ & 0.7 \\
\hline NGC 6569 & 7 & $4.62 \pm 0.19$ & $-0.91 \pm 0.14$ & $-1.02 \pm 0.16$ & $-1.03 \pm 0.21$ & $-0.66 \pm 0.07$ & -0.76 & 4 & $-0.72 \pm 0.14$ & 0.8 \\
\hline NGC 6656 & 41 & $3.12 \pm 0.30$ & $-1.74 \pm 0.11$ & $-1.81 \pm 0.13$ & $-1.74 \pm 0.20$ & $-1.92 \pm 0.02$ & -1.70 & 8 & $-1.70 \pm 0.08$ & 2.3 \\
\hline NGC 6715 & 15 & $3.30 \pm 0.25$ & $-1.65 \pm 0.11$ & $-1.74 \pm 0.13$ & $-1.66 \pm 0.20$ & $-1.31 \pm 0.04^{a}$ & -1.49 & 7 & $-1.44 \pm 0.07$ & 2.5 \\
\hline Terzan 7 & 7 & $5.62 \pm 0.12$ & $-0.21 \pm 0.12$ & $-0.19 \pm 0.13$ & $-0.53 \pm 0.20$ & $-0.23 \pm 0.10^{a}$ & -0.32 & 6 & $-0.12 \pm 0.08$ & 0.4 \\
\hline NGC 7006 & 18 & $3.49 \pm 0.25$ & $-1.56 \pm 0.11$ & $-1.65 \pm 0.13$ & $-1.57 \pm 0.20$ & $-1.69 \pm 0.09$ & -1.52 & 6 & $-1.46 \pm 0.06$ & 1.2 \\
\hline
\end{tabular}

Notes. Columns as in Table 4. The average reduced EW in Col.3 are from Saviane et al. (2012). ${ }^{(a)}$ Metallicities from Dias et al. (2016b).

members after applying our selection criteria mainly driven by radial velocities. Our selection is consistent with the spatial distribution of the stars since all the rejected objects are located far from the cluster centre. From the member stars we obtained a mean metallicity $[\mathrm{Fe} / \mathrm{H}]_{\mathrm{S} 12}=-1.48$, consistent with the value derived from near-IR photometry (Valenti et al. 2010). We also confirm the exceptionally high radial velocity for a globular cluster $\left(-358 \mathrm{~km} \mathrm{~s}^{-1}\right)$. Given the low galactic longitude and latitude of this cluster, the velocity is also high in the Galactic rest frame. Depending on the tangential velocity (proper motion), this might approach a space velocity close to the escape velocity from the Galaxy.

\subsection{Djorgovski 2}

The position of Djorg 2 (also known as ESO456-SC38) close to the Galactic centre, $(l, b)=(2.7,-2.5)$ and $R_{\mathrm{GC}}=1.8 \mathrm{kpc}$, makes extracting a clean CMD difficult because of crowding and high reddening $[E(B-V)=0.94]$. From NTT CMDs in the $V, I$ bands, Ortolani et al. (1997) found a metallicity $[\mathrm{Fe} / \mathrm{H}] \sim-0.5$, while the near-infrared study of Valenti et al. $(2010)$ gave $[\mathrm{Fe} / \mathrm{H}]=$ -0.65 , in both cases from the RGB morphology. Rossi et al. (2015) made an attempt to decontaminate the CMD using twoepoch observations and proper motions, which however did not give conclusive results because of a high differential reddening and the loose nature of the cluster.

As was the case with Djorg 1, our selection criteria gave only three member stars with a mean radial velocity $v_{\mathrm{r}} \approx-160 \mathrm{~km} \mathrm{~s}^{-1}$, consistent with the velocity measured from our optical spectra (Dias et al. 2016a), $v_{\mathrm{r}}=-150 \pm 28 \mathrm{~km} \mathrm{~s}^{-1}$. The derived mean metallicity is $[\mathrm{Fe} / \mathrm{H}]_{\mathrm{S} 12}=-1.09$, about 0.5 dex more metal-poor than the photometric determinations. The metallicity from the optical region is $[\mathrm{Fe} / \mathrm{H}]_{\mathrm{D} 16}=-0.79$, which is $\sim 0.3$ dex more metal rich than our present $\mathrm{CaT}$ estimate.

The optical CMD of Djorg 2 obtained from our pre-imaging observations is shown in Fig. 7 together with two clusters with similar metallicity, NGC 6401 and NGC 6864. For each cluster we plot the photometry obtained from the inner cluster region $(r<0.5$ arcmin), on top of the full field photometry. Over-plotted in each panel is the fiducial line published by Catelan et al. (2002) for NGC 6864, which is helpful to compare the RGB and HB morphology. Given the little number of confirmed cluster members, this CMD does not discriminate between a higher or lower metallicity. A larger star sample and possibly high-resolution spectroscopy will be useful to pinpoint the metallicity of this cluster.

\subsection{NGC 6256}

This globular cluster is located in the outer bulge at $(l, b)=$ $(-12.21,3.31)$ with a distance from the Galactic centre $R_{\mathrm{GC}}=$ $3.0 \mathrm{kpc}$. The high extinction of this cluster, $E(B-V)=1.01$, prevented so far spectroscopic studies of individual stars in the visible domain. Ortolani et al. (1999) analysed the optical VI CMD, finding evidence of an extended blue horizontal branch (BHB) similar to other intermediate metallicity clusters with metallicity close to $[\mathrm{Fe} / \mathrm{H}]=-1.5$. Integrated spectra from Bica et al. (1998) give a metallicity $[\mathrm{Fe} / \mathrm{H}]=-1.01$. HST data analysis by Piotto et al. (2002) gave a $B V$ CMD (with a large photometric scatter) confirming the extended blue $\mathrm{HB}$. The adopted metallicity was relatively high, $[\mathrm{Fe} / \mathrm{H}]=-0.70$. A lower metallicity is supported by the studies of Stephens \& Frogel(2004) and Valenti et al. (2007), who found $[\mathrm{Fe} / \mathrm{H}]=-1.35$ and $[\mathrm{Fe} / \mathrm{H}]=-1.63$, respectively. The Stephens \& Frogel (2004) measurements were performed on $\mathrm{Na}, \mathrm{Ca}$, and $\mathrm{CO}$ features using medium-resolution spectroscopy in the $2.2 \mu \mathrm{m}$ spectral region, while the Valenti et al. (2007) value was obtained from near-IR photometry.

We classified ten stars as cluster members, mostly because of their radial velocity, with only one star rejected because of its line strength. The mean radial velocity is consistent with previous estimates $\left(\Delta v_{\mathrm{r}}=-2.0 \mathrm{~km} \mathrm{~s}^{-1}\right.$ relative to H10). From those ten stars, the CaT line strengths favour a low metallicity, $[\mathrm{Fe} / \mathrm{H}]_{\mathrm{S} 12}=-1.61$. A high $\rho$ index $(\rho=2.2$, the highest of 
the sample) suggests an intrinsic metallicity dispersion $\sigma_{[\mathrm{Fe} / \mathrm{H}]} \approx$ 0.2 dex, for which we plan a follow-up study.

\subsection{NGC 6355}

This is another bulge globular cluster located $1.4 \mathrm{kpc}$ from the Galactic centre, in a relatively high-extinction area $[E(B-V)=$ 0.77] behind a dark nebula in the eastern part of the Ophiuchus complex. H10 and C09 used the Zinn \& West (1984) analysis of integrated light as a reference metallicity, obtaining a mean metallicity $[\mathrm{Fe} / \mathrm{H}] \approx-1.35$. More recent estimates are from photometric studies in the optical (Ortolani et al. 2003) and nearinfrared (Valenti et al. 2007) which suggest a metal poor value $[\mathrm{Fe} / \mathrm{H}]=-1.6$, once transformed to the $\mathrm{C} 09$ metallicity scale. Our metallicity measurement from CaT is $[\mathrm{Fe} / \mathrm{H}]_{\mathrm{S} 12}=-1.59$, while Dias et al. (2016a) found $[\mathrm{Fe} / \mathrm{H}]_{\mathrm{D} 16}=-1.46$.

\subsection{NGC 6366}

This cluster has been catalogued as either a bulge or a halo globular cluster (Campos et al. 2013). It is located at $3.5 \mathrm{kpc}$ from the Sun ( $5 \mathrm{kpc}$ from the Galactic centre) and $1 \mathrm{kpc}$ above the plane. Its radial velocity $\left(v_{\mathrm{r}}=-118 \mathrm{~km} \mathrm{~s}^{-1}\right)$ is consistent with halo kinematics as pointed out by Da Costa \& Seitzer (1989), who found $v_{\mathrm{r}}=-123 \mathrm{~km} \mathrm{~s}^{-1}$. The previous metallicity estimate listed in appendix 1 of C09, based on the CaT method applied by Da Costa \& Armandroff (1995) and Da Costa \& Seitzer (1989), agrees with the value measured by Dias et al. (2016a) from optical spectra, $[\mathrm{Fe} / \mathrm{H}]_{\mathrm{D} 16}=-0.61$. This value has recently been confirmed by detailed abundance analysis of eight red giant stars (Puls et al. 2018) which gave $[\mathrm{Fe} / \mathrm{H}]=-0.60$ and a mean radial velocity $v_{\mathrm{r}}=-121.2 \mathrm{~km} \mathrm{~s}^{-1}$. Our present CaT estimate is $[\mathrm{Fe} / \mathrm{H}]_{\mathrm{S} 12}=-0.81 \pm 0.14$ (from 14 stars), that is $\sim 0.2$ dex less metal rich yet consistent within the error.

The photometric study of Monelli et al. (2013) suggested a bimodality on the red giant branch. We measured a relatively large $\sigma_{[\mathrm{Fe} / \mathrm{H}]}(\rho=1.3)$ which marginally suggests an intrinsic metallicity dispersion. However, Puls et al. (2018) did not found any significant star-to-star abundance scatter. The projected radial distributions of stellar metallicities and radial velocities do not show any systematics with distance from the cluster centre.

\subsection{NGC 6401}

This globular cluster is located in the inner bulge region at $\sim 0.8 \mathrm{kpc}$ from the Galactic centre, with extinction $E(B-V)=$ 0.72. From optical photometry Barbuy et al. (1999) characterised this cluster as metal-rich, with metallicity similar to that of NGC $104([\mathrm{Fe} / \mathrm{H}]=-0.70)$. Later, the optical and NIR photometric studies of Piotto et al. (2002) and Valenti et al. (2007) detected a blue extended HB consistent with a more metal poor value $[\mathrm{Fe} / \mathrm{H}]=-1.56$. The presence of a blue $\mathrm{HB}$ is also confirmed by our optical photometry from the pre-imaging, and consistent with the metallicity derived here from seven cluster members $\left([\mathrm{Fe} / \mathrm{H}]_{\mathrm{S} 12}=-1.30\right)$. The metallicity obtained from optical spectra is $[\mathrm{Fe} / \mathrm{H}]_{\mathrm{D} 16}=-1.12$, in agreement with the only previous spectroscopic study of individual stars $([\mathrm{Fe} / \mathrm{H}]=-1.1$, Minniti 1995).

Minniti (1995) obtained a radial velocity $-78 \pm 33 \mathrm{~km} \mathrm{~s}^{-1}$ while our value is $v_{\mathrm{r}}=-115 \mathrm{~km} \mathrm{~s}^{-1}$, in agreement with the result of Dias et al. (2016a), $v_{\mathrm{r}}=-120 \pm 17 \mathrm{~km} \mathrm{~s}^{-1}$. This discrepancy is possibly related to field contamination in the Minniti
(1995) sample. From the Besançon model (Robin et al. 2003) the typical radial velocity for this field is $v_{\mathrm{r}} \approx+60 \mathrm{~km} \mathrm{~s}^{-1}$ with a broad distribution $\left(\sigma_{v_{\mathrm{r}}} \approx 110 \mathrm{~km} \mathrm{~s}^{-1}\right)$. These values are consistent with the velocity distribution of stars rejected from our sample, located at $r>2$ arcmin from the cluster centre.

\subsection{NGC 6426}

This metal-poor halo globular cluster is located at $21 \mathrm{kpc}$ from the Sun in a relatively low-extinction area $[E(B-V)=$ $0.39]$. The $\mathrm{H} 10$ catalogue lists a metallicity $[\mathrm{Fe} / \mathrm{H}]=-2.15$ based on integrated spectra (ZW84). From CMD photometry, Hatzidimitriou et al. (1999) measured a metallicity $[\mathrm{Fe} / \mathrm{H}]=$ $-2.33 \pm 0.15$ and an age marginally older than M92. Such a low metallicity has been confirmed by Dias et al. (2015) $\left([\mathrm{Fe} / \mathrm{H}]_{\mathrm{D} 16}=-2.39\right)$ and more recently by high-resolution spectroscopy of four red giants by Hanke et al. (2017). Their detailed abundance analysis gives a mean $\mathrm{Fe}$ abundance $[\mathrm{Fe} / \mathrm{H}]=$ $-2.34 \pm 0.05$ dex with a mean $\alpha$-elements-to-Fe ratio of $0.39 \pm$ 0.03 . We classified here seven stars as cluster members from a relatively easy selection process. The target stars are clearly split in two groups: one with large negative radial velocities and the other close to zero. This second group has radial velocities consistent with the Besançon model predictions (Robin et al. 2003), as shown in Sharina et al. (2012). For the members we found a mean radial velocity $v_{\mathrm{r}}=-220 \mathrm{~km} \mathrm{~s}^{-1}$ which is more negative than the value published in $\mathrm{H} 10\left(v_{\mathrm{r}}=-162 \mathrm{~km} \mathrm{~s}^{-1}\right)$ and close to the result of Hanke et al. (2017) from high-resolution spectroscopy $\left(-212.2 \pm 0.5 \mathrm{~km} \mathrm{~s}^{-1}\right)$. We measured a (slightly extrapolated) mean metallicity $[\mathrm{Fe} / \mathrm{H}]_{\mathrm{S} 12}=-2.38$ for the cluster stars, which is the lowest value in our sample and is consistent with the results of Dias et al. (2015) and Hanke et al. (2017).

\subsection{NGC 6517}

For this cluster, Kavelaars et al. (1995) found a metallicity $[\mathrm{Fe} / \mathrm{H}]=-1.58 \pm 0.05$ from $B V I$ photometry after correction for differential reddening. Previous optical low-resolution spectroscopy of individual stars by Minniti (1995) gave a metallicity $[\mathrm{Fe} / \mathrm{H}]=-1.20$, close to the value derived from integrated light by $\mathrm{ZW} 84,[\mathrm{Fe} / \mathrm{H}]=-1.34 \pm 0.15$. From nine member stars we found $[\mathrm{Fe} / \mathrm{H}]_{\mathrm{S} 12}=-1.63$, a value significantly more metal-poor than the metallicity tabulated by $\mathrm{C} 09$, which has its origin in the Minniti (1995) and ZW84 measurements.

\subsection{NGC 6864}

NGC 6864 (M 75) is an outstanding globular cluster located $15 \mathrm{kpc}$ away from the Galactic centre in the interface region between the inner and outer Galactic halo (Zinn 1993; Carollo et al. 2007). As one of the most massive and centrally concentrated GCs in the Milky Way (Harris 1996), it contrasts with the typical characteristics of size and low concentration of GCs in the outer halo (Koch et al. 2009; Koch \& Côté 2010). Its relatively high metallicity derived from high-resolution spectroscopy $([\mathrm{Fe} / \mathrm{H}]=-1.16$, Kacharov et al. 2013), together with an age of $\sim 10 \mathrm{Gyr}$ (Catelan et al. 2002), have suggested a possible extragalactic origin. The spectroscopic study of Kacharov et al. (2013) found three generations of stars apparently formed on a short timescale, more closely associated with an extended $\mathrm{Na}-\mathrm{O}$ anticorrelation than an $\mathrm{Fe}$ abundance scatter. The right panel of Fig. 7 shows an optical CMD derived from our pre-imaging photometry, confirming the HB morphology discussed by Catelan et al. (2002), characterised by a tri-modal HB. 
S. Vásquez et al.: Homogeneous metallicities and radial velocities for Galactic globular clusters. II.

From our measurements we estimate a cluster metallicity $[\mathrm{Fe} / \mathrm{H}]_{\mathrm{S} 12}=-1.14$, consistent with the estimate of Dias et al. (2016a) $\left([\mathrm{Fe} / \mathrm{H}]_{\mathrm{D} 16}=-1.00\right)$ and with previous measurements. A $\rho$ index of 1.3 suggests a moderate metallicity spread from 11 member stars. This metallicity scatter, if not caused by a mix of stellar populations as suggested by Kacharov et al. (2013), can be related to the high luminosity, meaning mass, of this cluster $\left(M_{V}=-8.57 \mathrm{mag}\right)$, in accord with the correlation between metallicity dispersion and cluster luminosity found by Carretta et al. (2009).

To directly compare our measurements with those of Kacharov et al. (2013) we looked for common targets, finding six stars. One of the six stars (labelled 1_9285 in our programme) was rejected as a non-member by our selection criteria due to its radial velocity $-168 \mathrm{~km} \mathrm{~s}^{-1}$. This value is $4 \sigma$ off with respect to the mean value measured from the 11 member stars $\left(v_{\mathrm{r}}=-193.4 \mathrm{~km} \mathrm{~s}^{-1}, \sigma_{v_{\mathrm{r}}}=5 \mathrm{~km} \mathrm{~s}^{-1}\right)$. From the remaining five stars in common we obtain mean values in good agreement: $[\mathrm{Fe} / \mathrm{H}]_{\mathrm{S} 12}=-1.13$ versus $[\mathrm{Fe} / \mathrm{H}]_{\mathrm{HR}}=-1.19$ (dispersion of the difference $\sim 0.01$ dex).

\subsection{Pal 10}

Pal 10 is a highly obscured globular cluster, with a reddening $E(B-V)=1.66$, located $0.3 \mathrm{kpc}$ above the Galactic plane and $6 \mathrm{kpc}$ away from the Sun. Its optical CMD shows significant effects from differential reddening, which introduces an uncertainty of the order $\sim 0.2$ mag on the HB location. A previous metallicity estimate comes from the optical photometry of Kaisler et al. (1997) who measured $[\mathrm{Fe} / \mathrm{H}]=-0.1$. Our analysis gives a somewhat less metal rich value from 9 members stars, $[\mathrm{Fe} / \mathrm{H}]_{\mathrm{S} 12}=$ -0.27 , to be compared to $[\mathrm{Fe} / \mathrm{H}]_{\mathrm{D} 16}=-0.24$ from spectral synthesis in the optical range. The calibration of Vásquez et al. (2015) yields $[\mathrm{Fe} / \mathrm{H}]_{\mathrm{V} 15}=-0.57$, illustrating the sensitivity to the different calibrations in the metal rich regime.

\subsection{Terzan 1}

The globular cluster Terzan 1 is located almost on the Galactic plane $(0.1 \mathrm{kpc}$ above it), in the inner region of the Galactic bulge at $1.3 \mathrm{kpc}$ from the Milky Way centre. Its reddening $E(B-V)=$ 1.99 is the highest in our sample, with important differential effects on the optical CMD. This cluster is considered one of the second-parameter globular clusters in the Galactic bulge (along with NGC 6388 and 6441), because of the presence of a red HB along with a relatively low metallicity. Previous metallicity estimates include measures from optical photometry $([\mathrm{Fe} / \mathrm{H}] \approx$ -1.2 , Ortolani et al. 1999), NIR photometry $([\mathrm{Fe} / \mathrm{H}]=-1.11$, Valenti et al. 2010), and low-resolution optical spectroscopy from Idiart et al. $(2002)([\mathrm{Fe} / \mathrm{H}] \approx-1.3)$. High-resolution near-infrared spectra of 15 stars in the inner region of Terzan 1 (Valenti et al. 2015 ) yield $[\mathrm{Fe} / \mathrm{H}]=-1.26 \pm 0.03 \mathrm{dex}$. Our selection provides 9 member stars, located in the inner region of the cluster $\left(r<2\right.$ arcmin), with a mean velocity $v_{\mathrm{r}}=63 \mathrm{~km} \mathrm{~s}^{-1}$ and dispersion $\sigma_{v_{\mathrm{r}}}=8 \mathrm{~km} \mathrm{~s}^{-1}$. This result is consistent with the result of Valenti et al. (2015), $v_{\mathrm{r}}=57 \mathrm{~km} \mathrm{~s}^{-1}$, while there is a difference $\Delta v_{\mathrm{r}}=-51 \mathrm{~km} \mathrm{~s}^{-1}$ from the mean radial velocity measured by Idiart et al. (2002). From the mean $W^{\prime}$ of member stars we derived a metallicity $[\mathrm{Fe} / \mathrm{H}]_{\mathrm{S} 12}=-0.84$, slightly more metal-rich than literature values and the Dias et al. (2016b) determination.

\subsection{Ton 2}

The globular cluster Ton 2 (Tonantzintla 2 ) is located in the central region of the Galactic bulge $(R=1.4 \mathrm{kpc}), 0.5 \mathrm{kpc}$ below the Galactic plane. Its metallicity and extinction derived from NTT optical photometry are $[\mathrm{Fe} / \mathrm{H}] \approx-0.6$ and $E(B-V)=$ 1.24, respectively (Bica et al. 1996). Its HB morphology suggests an important differential reddening similar to that of Pal 10, leading to an uncertainty of $0.22 \mathrm{mag}$ in the $\mathrm{HB}$ magnitude. No previous spectroscopic observations exist for this cluster. The CaT reduced equivalent width implies a high metallicity $[\mathrm{Fe} / \mathrm{H}]_{\mathrm{S} 12}=-0.26$, which is also close to that of Pal 10. This value is more metal-rich than the values tabulated by $\mathrm{H} 10$ and Dias et al. (2016b), which originate from the CMD-based [Fe/H] determination of Bica et al. (1996).

\subsection{Terzan 8}

Terzan 8 is a globular cluster in the Milky Way halo associated with the Sgr dwarf galaxy (Da Costa \& Armandroff 1995; Montegriffo et al. 1998; Law \& Majewski 2010). The extinction along the line of sight is low, $E(B-V)=0.12$. This cluster has previous measurements from high-resolution spectroscopy by Mottini et al. (2008) and Carretta et al. (2014), giving metallicities $[\mathrm{Fe} / \mathrm{H}]=-2.35 \pm 0.05$ and $[\mathrm{Fe} / \mathrm{H}]=-2.27 \pm 0.08$, respectively. From our measurements, we obtained $[\mathrm{Fe} / \mathrm{H}]_{\mathrm{S} 12}=-2.15$, close to the results of high-resolution spectroscopy. From the optical spectra the mean metallicity is $[\mathrm{Fe} / \mathrm{H}]_{\mathrm{D} 16}=-2.06$, in agreement with our $\mathrm{CaT}$ results within $1 \sigma$.

\section{Summary and discussion}

We have obtained new radial velocities and metallicities for stars in 28 Milky Way GCs, as the completion of our spectroscopic survey aimed at providing a homogeneous set of metallicities from medium resolution data in two spectral regions. The results in this paper are based on FORS2 spectroscopy with a resolution $R \sim 2500$ in the region centred on the Ca II triplet lines (8450-8700 $\AA$ ) of individual cluster candidate red giants. In a previous study of this series, Saviane et al. (2012) measured the metallicity of 20 programme clusters using eight template clusters with known metallicity to establish an empirical CaT line strength calibration. In this paper we have measured the metallicity of an additional sample of 28 globular clusters mainly in the Galactic bulge or far in the halo. Most of the clusters analysed here are therefore faint and/or highly reddened, which for a long time prevented any metallicity measurement based on spectroscopy of individual stars. We provide metallicities based on the CaT calibration of Saviane et al. (2012), with typical uncertainties of the order $\sim 0.15$ dex. We also discussed the impact on the measured $[\mathrm{Fe} / \mathrm{H}]$ of alternative empirical calibrations such as those of Vásquez et al. (2015) and Da Costa (2016), with differences especially noticeable in the metal-rich regime $([\mathrm{Fe} / \mathrm{H}]>-0.8)$. Our sample is complemented by the 71 GCs published by Rutledge et al. (1997) transformed to our metallicity scale (Saviane et al. 2012). Altogether, our project provides the largest GC sample with homogeneous metallicity measurements from CaT currently available. The combination of all data yields CaT metallicity estimates on the same scale for $107 \mathrm{GCs}$, that is $\sim 69 \%$ of the clusters published in $\mathrm{H} 10$. The metallicity distribution of all clusters in our CaT project is plotted in Fig. 9, where the new data represent about $50 \%$ of the available measurements for metal-rich clusters. As a test of the metallicity scale provided by the Ca II triplet, we compared our results with those obtained from spectroscopy of stars in the same clusters using a spectral fitting technique in the optical region (Dias et al. 2015, 2016a). The comparison shows that the 


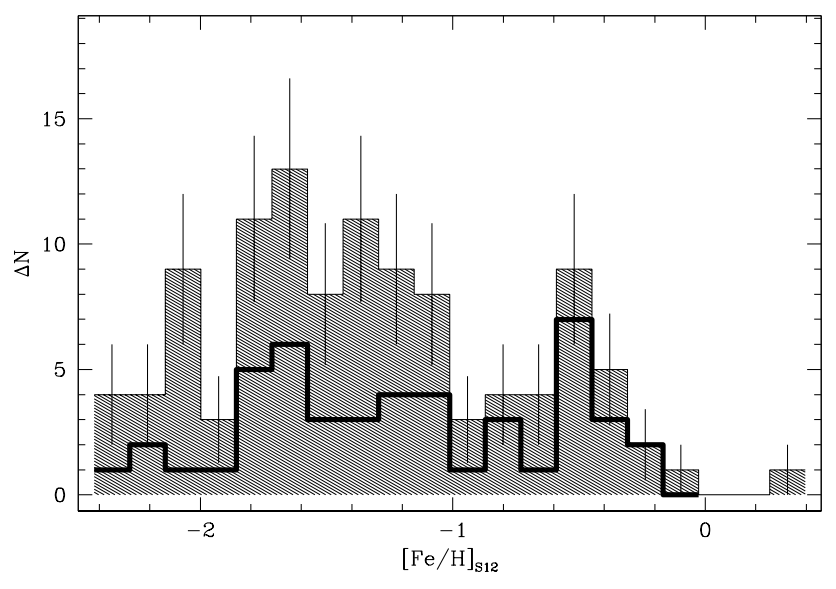

Fig. 9. Metallicity distribution of the MW GCs in the merged CaT sample. The thick line shows the histogram made with our data only (Paper I and the present work); the remaining data are from Rutledge et al. (1997) as put on the C09 scale by Saviane et al. (2012). The error bars are Poisson errors on the number of clusters in each bin.

metallicities measured from the CaT line strengths using the calibration of Saviane et al. (2012) are consistent within the errors with the $[\mathrm{Fe} / \mathrm{H}]$ values derived from spectral fitting by Dias et al., which in turn agree with metal abundances from high-resolution spectroscopy. Our study confirms the CaT method as a powerful tool to determine $[\mathrm{Fe} / \mathrm{H}]$ for stellar systems so distant or reddened that high-resolution spectroscopic analysis becomes very difficult or prohibitive, provided that the calibrators are selected from the same population as the targets. This will be particularly useful to study resolved stars beyond the Local Group with the new generation of extremely large telescopes.

We have identified some interesting cases among the $28 \mathrm{GCs}$ analysed in the present work. We obtained a much improved metallicity measurement for Djorg 2: while previous work based on a NIR CMD classified it as a metal-rich globular cluster with a metallicity $[\mathrm{Fe} / \mathrm{H}]=-0.65$, our EW measurements and optical photometry suggest a more metal-poor value $[\mathrm{Fe} / \mathrm{H}] \approx-1.1$. Additionally, we found a radial velocity $v_{\mathrm{r}}=-160 \mathrm{~km} \mathrm{~s}^{-1}$ which is the first estimate for this cluster. Another interesting case is that of Terzan 1, for which we found a difference $\Delta v_{\mathrm{r}}=-51 \mathrm{~km} \mathrm{~s}^{-1}$ in radial velocity with respect to previous values. Our estimate is consistent with the latest measurement from high-resolution spectra (Valenti et al. 2015). Finally, as in Saviane et al. (2012), we explored the presence of intrinsic metallicity dispersions within our sample: we found three clusters that might have a metallicity dispersion, based on a comparison of their EW dispersion with the intrinsic error in EW measurements (parameter $\rho \geqslant 1.3$ ). One of them, NGC 6256, is the most probable candidate, with $\rho=2.2$. For this cluster our data suggest an intrinsic metallicity dispersion $\sigma_{[\mathrm{Fe} / \mathrm{H}]}=0.2 \mathrm{dex}$, but more data are needed to confirm this hint.

Acknowledgements. The authors thank the anonymous referee for helpful comments and suggestions. SV and MZ acknowledge support by the Ministry of Economy, Development, and Tourism's Millennium Science Initiative through grant IC120009, awarded to The Millennium Institute of Astrophysics (MAS), by Fondecyt Regular 1150345 and by the BASAL-CATA Center for Astrophysics and Associated Technologies PFB-06. SV also acknowledges support by the ESO studentship programme 2012-2014.

\section{References}

Armandroff, T. E., \& Da Costa, G. S. 1991, AJ, 101, 1329 Armandroff, T. E., \& Zinn, R. 1988, AJ, 96, 92
Barbuy, B., Ortolani, S., Bica, E., \& Desidera, S. 1999, A\&A, 348, 783

Battaglia, G., \& Starkenburg, E. 2012, A\&A, 539, A123

Baumgardt, H., \& Hilker, M. 2018, MNRAS, 478, 1520

Bica, E., Ortolani, S., \& Barbuy, B. 1996, A\&AS, 120, 153

Bica, E., Claria, J. J., Piatti, A. E., \& Bonatto, C. 1998, A\&AS, 131, 483

Campos, F., Kepler, S. O., Bonatto, C., \& Ducati, J. R. 2013, MNRAS, 433, 243

Carollo, D., Beers, T. C., Lee, Y. S., et al. 2007, Nature, 450, 1020

Carrera, R., Pancino, E., Gallart, C., \& del Pino, A. 2013, MNRAS, 434 1681

Carretta, E., Bragaglia, A., Gratton, R., D’Orazi, V., \& Lucatello, S. 2009, A\&A, 508,695

Carretta, E., Bragaglia, A., Gratton, R. G., et al. 2014, A\&A, 561, A87

Catelan, M., Borissova, J., Ferraro, F. R., et al. 2002, AJ, 124, 364

Cohen, R. E., Moni Bidin, C., Mauro, F., Bonatto, C., \& Geisler, D. 2017, MNRAS, 464, 1874

Cole, A. A., Smecker-Hane, T. A., Tolstoy, E., Bosler, T. L., \& Gallagher, J. S. 2004, MNRAS, 347, 367

Da Costa, G. S. 2016, MNRAS, 455, 199

Da Costa, G. S., \& Armandroff, T. E. 1995, AJ, 109, 2533

Da Costa, G. S., \& Seitzer, P. 1989, AJ, 97, 405

Da Costa, G. S., Held, E. V., \& Saviane, I. 2014, MNRAS, 438, 3507

Davoust, E., Sharina, M. E., \& Donzelli, C. J. 2011, A\&A, 528, A70

Dias, B., Barbuy, B., Saviane, I., et al. 2015, A\&A, 573, A13

Dias, B., Barbuy, B., Saviane, I., et al. 2016a, A\&A, 590, A9

Dias, B., Saviane, I., Barbuy, B., et al. 2016b, The Messenger, 165, 19

Frinchaboy, P. M., Muñoz, R. R., Phelps, R. L., Majewski, S. R., \& Kunkel, W. E. 2006, AJ, 131, 922

Gratton, R. G., Carretta, E., \& Bragaglia, A. 2012, A\&ARv, 20, 50

Gullieuszik, M., Held, E. V., Saviane, I., \& Rizzi, L. 2009, A\&A, 500, 735

Hanke, M., Koch, A., Hansen, C. J., \& McWilliam, A. 2017, A\&A, 599, A97

Harris, W. E. 1996, AJ, 112, 1487

Harris, W. E. 2010, ArXiv e-prints [arXiv:1012. 3224]

Hatzidimitriou, D., Papadakis, I., Croke, B. F. W., et al. 1999, AJ, 117, 3059

Horne, K. 1986, PASP, 98, 609

Idiart, T. P., Barbuy, B., Perrin, M.-N., et al. 2002, A\&A, 381, 472

Kacharov, N., Koch, A., \& McWilliam, A. 2013, A\&A, 554, A81

Kaisler, D., Harris, W. E., \& McLaughlin, D. E. 1997, PASP, 109, 920

Kavelaars, J. J., Hanes, D. A., Bridges, T. J., \& Harris, W. E. 1995, AJ, 109, 2081

Koch, A., \& Côté, P. 2010, A\&A, 517, A59

Koch, A., Côté, P., \& McWilliam, A. 2009, A\&A, 506, 729

Law, D. R., \& Majewski, S. R. 2010, ApJ, 718, 1128

Minniti, D. 1995, A\&A, 303, 468

Monelli, M., Milone, A. P., Stetson, P. B., et al. 2013, MNRAS, 431, 2126

Montegriffo, P., Bellazzini, M., Ferraro, F. R., et al. 1998, MNRAS, 294, 315

Mottini, M., Wallerstein, G., \& McWilliam, A. 2008, AJ, 136, 614

Ortolani, S., Bica, E., \& Barbuy, B. 1995a, A\&A, 300, 726

Ortolani, S., Bica, E., \& Barbuy, B. 1995b, A\&A, 296, 680

Ortolani, S., Bica, E., \& Barbuy, B. 1997, A\&AS, 126, 319

Ortolani, S., Barbuy, B., \& Bica, E. 1999, A\&AS, 136, 237

Ortolani, S., Bica, E., \& Barbuy, B. 2003, A\&A, 402, 565

Phelps, R. L., \& Schick, M. 2003, AJ, 126, 265

Piotto, G., King, I. R., Djorgovski, S. G., et al. 2002, A\&A, 391, 945

Pryor, C., \& Meylan, G. 1993, ASP Conf. Ser., 50, 357

Puls, A. A., Alves-Brito, A., Campos, F., Dias, B., \& Barbuy, B. 2018, MNRAS, 476, 690

Robin, A. C., Reylé, C., Derrière, S., \& Picaud, S. 2003, A\&A, 409, 523

Rossi, L. J., Ortolani, S., Barbuy, B., Bica, E., \& Bonfanti, A. 2015, MNRAS, 450, 3270

Rutledge, G. A., Hesser, J. E., \& Stetson, P. B. 1997, PASP, 109, 907

Saviane, I., da Costa, G. S., Held, E. V., et al. 2012, A\&A, 540, A27

Sharina, M., Aringer, B., Davoust, E., Kniazev, A. Y., \& Donzelli, C. J. 2012 MNRAS, 426, L31

Sharina, M. E., Donzelli, C. J., Davoust, E., Shimansky, V. V., \& Charbonnel, C. 2014, A\&A, 570, A48

Starkenburg, E., Hill, V., Tolstoy, E., et al. 2010, A\&A, 513, A34

Stephens, A. W., \& Frogel, J. A. 2004, AJ, 127, 925

Stetson, P. B. 1987, PASP, 99, 191

Stetson, P. B. 1994, PASP, 106, 250

Valenti, E., Ferraro, F. R., \& Origlia, L. 2007, AJ, 133, 1287

Valenti, E., Ferraro, F. R., \& Origlia, L. 2010, MNRAS, 402, 1729

Valenti, E., Origlia, L., Mucciarelli, A., \& Rich, R. M. 2015, A\&A, 574, A80

Vásquez, S., Zoccali, M., Hill, V., et al. 2015, A\&A, 580, A121

Warren, S. R., \& Cole, A. A. 2009, MNRAS, 393, 272

Zinn, R., \& West, M. J. 1984, ApJS, 55, 45

Zinn, R., 1993, ASP Conf. Ser., 48, 38 